\title{
RIEMANNIAN EXPONENTIAL MAPS OF THE DIFFEOMORPHISM GROUP OF $\mathbb{T}^{2 *}$
}

\author{
THOMAS KAPPELER ${ }^{\dagger}$, ENRIQUE LOUBET $^{\ddagger}$, AND PETER TOPALOV $^{\S}$
}

\begin{abstract}
We study the exponential maps induced by right-invariant weak Riemannian metrics of Sobolev type of order $k \geq 0$ on the Lie group of smooth, orientation preserving diffeomorphisms of the two dimensional torus. We prove that for $k \geq 1$, but not for $k=0$, each of them defines a smooth Fréchet chart of the identity.
\end{abstract}

Key words. Geodesic exponential maps, diffeomorphism group of the torus

AMS subject classifications. 58D05, 58E10

1. Introduction. The aim of this paper is to contribute towards a theory of Riemannian geometry on infinite dimensional Lie groups. These groups have attracted a lot of attention since Arnold's seminal paper [1] on hydrodynamics - e.g. [12], [19], [24], [25]. As a case study we consider the Lie group $\mathcal{D}_{+}=\mathcal{D}_{+}\left(\mathbb{T}^{2}\right)$ of orientation preserving $C^{\infty}$-diffeomorphisms of the two dimensional torus $\mathbb{T}^{2}=\mathbb{R}^{2} / \mathbb{Z}^{2}$. The Lie algebra $T_{\mathrm{id}} \mathcal{D}_{+}$of $\mathcal{D}_{+}$is the space $C^{\infty}\left(\mathbb{T}^{2}, \mathbb{R}^{2}\right)$ of smooth vector fields on $\mathbb{T}^{2}$. We remark that $\mathcal{D}_{+}$and its Lie algebra come up in hydrodynamics playing the role of configuration spaces for compressible and inviscid fluids on $\mathbb{T}^{2}$.

For any given $k \geq 0$, consider the scalar product $\langle\cdot, \cdot\rangle_{k}: C^{\infty}\left(\mathbb{T}^{2}, \mathbb{R}^{2}\right) \times$ $C^{\infty}\left(\mathbb{T}^{2}, \mathbb{R}^{2}\right) \rightarrow \mathbb{R}$

$$
\langle u, v\rangle_{k}:=\sum_{0 \leq j \leq k} \int_{\mathbb{T}^{2}}\left\langle(-\Delta)^{j} u, v\right\rangle d x
$$

where $\langle\cdot, \cdot\rangle$ denotes the Euclidean scalar product in $\mathbb{R}^{2}$. It induces a $C_{F}^{1}$-smooth weak right-invariant Riemannian metric $\nu^{(k)}$ on $\mathcal{D}_{+}$,

$$
\nu_{\varphi}^{(k)}(\xi, \eta):=\left\langle\left(d_{\mathrm{id}} R_{\varphi}\right)^{-1} \xi,\left(d_{\mathrm{id}} R_{\varphi}\right)^{-1} \eta\right\rangle_{k}, \quad \forall \varphi \in \mathcal{D}_{+}, \quad \text { and } \quad \forall \xi, \eta \in T_{\varphi} \mathcal{D}_{+}
$$

where $R_{\varphi}: \mathcal{D}_{+} \rightarrow \mathcal{D}_{+}, \psi \mapsto \psi \circ \varphi$ denotes the right translation by $\varphi$. The subscript $F$ in $C_{F}^{1}$, refers to the calculus in Fréchet spaces - see Appendix A where we collect some definitions and notions of the calculus in Fréchet spaces. The metric $\nu^{(k)}$ being weak means that the topology induced by $\nu^{(k)}$ on the tangent space $T_{\varphi} \mathcal{D}_{+}$at an arbitrary point $\varphi$ in $\mathcal{D}_{+}$, is weaker than the Fréchet topology on $T_{\varphi} \mathcal{D}_{+} \cong C^{\infty}\left(\mathbb{T}^{2}, \mathbb{R}^{2}\right)$ - see e.g. [12].

Definition 1.1. For any given $T>0$, a $C_{F}^{2}$-smooth curve $\varphi:[0, T] \rightarrow \mathcal{D}_{+}$, is called a geodesic for $\nu^{(k)}$, or $\nu^{(k)}$-geodesic for short, if it is a critical point of the

${ }^{*}$ Received August 4, 2005; accepted for publication May 2, 2008.

$\dagger$ Institut für Mathematik, Universität Zürich, Winterthurerstrasse 190, CH-8057 Zürich, Switzerland (thomas.kappeler@math.uzh.ch). Supported in part by the Swiss National Science Foundation, the programme SPECT, and the European Community through the FP6 Marie Curie RTN ENIGMA (MRTN-CT-2004-5652).

${ }^{\ddagger}$ Institut für Mathematik, Universität Zürich, Winterthurerstrasse 190, CH-8057 Zürich, Switzerland (eloubet@math.uzh.ch). Partially supported by the Swiss National Science Foundation.

$\S$ Department of Mathematics, Northeastern University, 360 Huntington Avenue, Boston, MA 02115, USA (p.topalov@neu.edu). 
action functional within the class of $C_{F}^{2}$-smooth variations $\gamma$ constrained to keep the end points fixed. More precisely, for any $C_{F}^{2}$-smooth function

$$
\gamma:(-\varepsilon, \varepsilon) \times[0, T] \rightarrow \mathcal{D}_{+}, \quad(s, t) \mapsto \gamma(s, t)
$$

satisfying $\gamma(0, t)=\varphi(t)$, for every $0 \leq t \leq T$, and $\gamma(s, 0)=\varphi(0)$ and $\gamma(s, T)=\varphi(T)$ for any $-\varepsilon<s<\varepsilon$, one has

$$
\left.\frac{d}{d s}\right|_{s=0} \mathcal{E}_{k}^{T}(\gamma(s, \cdot))=0
$$

where $\mathcal{E}_{k}^{T}$ denotes the action functional

$$
\mathcal{E}_{k}^{T}(\gamma(s, \cdot)):=\frac{1}{2} \int_{0}^{T} \nu_{\gamma(s, t)}^{(k)}(\dot{\gamma}(s, t), \dot{\gamma}(s, t)) d t,
$$

and $\dot{\gamma}(s, t)=\partial \gamma(s, t) / \partial t$.

The Euler-Lagrange equations for the action functional $\mathcal{E}_{k}^{T}$ defined in (1.1b) lead to the following initial value problem

$$
\left\{\begin{array}{l}
\dot{\varphi}=v, \quad \dot{v}=F_{k}(\varphi, v) \\
\varphi(0)=\mathrm{id}, \quad v(0)=v_{0}
\end{array}\right.
$$

where

$$
F_{k}(\varphi, v):=R_{\varphi} \circ A_{k}^{-1} \circ B_{k}\left(v \circ \varphi^{-1}\right)
$$

in which

$$
A_{k}:=\mathrm{Id}+\sum_{i=1}^{k}(-\Delta)^{i}
$$

and, for any smooth function $u$ in $C^{\infty}\left(\mathbb{T}^{2}, \mathbb{R}^{2}\right)$,

$$
B_{k}(u):=A_{k}((d u) u)-\left(d A_{k} u\right) u-\left(\operatorname{div} u \cdot \operatorname{Id}+(d u)^{\dagger}\right) A_{k} u .
$$

Here $(\cdot)^{\cdot}$ stands for $d / d t, \Delta=\partial_{x_{1}}^{2}+\partial_{x_{2}}^{2}$ is the Laplacian, $\left((d u)^{\dagger}\right)_{i j}=(d u)_{j i}$ the transpose of $d u$, and Id the $2 \times 2$ identity matrix. We remark that one can write $\left(d A_{k} u\right) u=(u \cdot \nabla) A_{k} u$, and $(d u) u=(u \cdot \nabla) u$, where $u \cdot \nabla$ is the vector field $u_{1} \partial_{x_{1}}+$ $u_{2} \partial_{x_{2}}$. The operators $u \cdot \nabla, \Delta$, and $A_{k}$, act componentwise on functions in $C^{\infty}\left(\mathbb{T}^{2}, \mathbb{R}^{2}\right)$. Note that $t \mapsto \varphi(t)$ evolves in $\mathcal{D}_{+}$whereas $t \mapsto \dot{\varphi}(t)=v(t)$ is a vector field along $\varphi$ i.e., a section of $\varphi^{*} T \mathcal{D}_{+}$, and $v(t)$ in $T_{\varphi(t)} \mathcal{D}_{+}$. It is easy to check that (1.2) is equivalent to the two initial value problems

$$
\left\{\begin{array}{l}
\dot{\varphi}=u \circ \varphi \\
\varphi(0)=\mathrm{id}
\end{array}\right.
$$

and

$$
\left\{\begin{array}{l}
\left(A_{k} u\right)^{\cdot}+\left(d A_{k} u\right) u+\left(\operatorname{div} u \cdot \operatorname{Id}+(d u)^{\dagger}\right) A_{k} u=0 \\
u(0)=v_{0}
\end{array}\right.
$$


where $t \mapsto u(t)=\left(d_{\text {id }} R_{\varphi(t)}\right)^{-1} \dot{\varphi}(t)$ is a curve in $T_{\text {id }} \mathcal{D}_{+}$. The initial value problems (1.2) and (1.7) are, via (1.6), two alternative descriptions of the geodesic flow. The first corresponds to the Lagrangian description i.e., tracking the flow as a section of $\varphi^{*} T \mathcal{D}_{+}$while the latter describes it in $T_{\text {id }} \mathcal{D}_{+}$from the Eulerian point of view of a fixed observer. For the convenience of the reader the derivation of (1.6)-(1.7) is reviewed in Appendix C.

For $k=0$, equation (1.7) can be viewed as a generalization (from one to two space dimensions) of the inviscid Burgers' equation. For $k=1$, the geodesic flow (1.2) is the analogue of the Camassa-Holm equation (1.7) in two space dimensions - see e.g. $[19],[20]$, and [25].

Our first result concerns the existence of the geodesic flow associated with the weak right-invariant Riemannian metric $\nu^{(k)}$ for any $k \geq 1$.

THEOREM 1.2. Let $k$ be an arbitrary integer in $\mathbb{Z}_{\geq 1}$. Then there exists an open neighborhood $V^{(k)}$ of 0 in $C^{\infty}\left(\mathbb{T}^{2}, \mathbb{R}^{2}\right)$ such that for any $v_{0}$ in $V^{(k)}$ there is a unique $\nu^{(k)}$-geodesic $(-2,2) \rightarrow \mathcal{D}_{+}, t \mapsto \varphi(t)$ issuing from the identity in the direction $v_{0}$ which depends $C_{F}^{1}$-smoothly on the initial data i.e., the map

$$
(-2,2) \times V^{(k)} \rightarrow \mathcal{D}_{+}, \quad\left(t, v_{0}\right) \mapsto \varphi\left(t ; v_{0}\right)
$$

is $C_{F}^{1}$-smooth.

Theorem 1.2 allows to define, for any given $k \geq 1$, the Riemannian exponential map

$$
\left.\operatorname{Exp}_{k}\right|_{V^{(k)}}: V^{(k)} \rightarrow \mathcal{D}_{+}, \quad v_{0} \mapsto \varphi\left(1 ; v_{0}\right)
$$

THEOREM 1.3. For any integer $k \geq 1$, there exist an open neighborhood $\tilde{V}^{(k)} \subseteq$ $V^{(k)}$ of 0 in $C^{\infty}\left(\mathbb{T}^{2}, \mathbb{R}^{2}\right)$ and an open neighborhood $U^{(k)}$ of id in $\mathcal{D}_{+}$so that

$$
\left.\operatorname{Exp}_{k}\right|_{\tilde{V}^{(k)}}: \tilde{V}^{(k)} \rightarrow U^{(k)}, \quad v_{0} \mapsto \varphi\left(1 ; v_{0}\right)
$$

is a $C_{F}^{1}$-diffeomorphism.

Most likely, Theorem 1.2 holds also for $k=0$ (even though, for this case, our method of proof does not work). However, Theorem 1.3 is false for $k=0$. Indeed, we construct a counter-example along the lines of $[7,8]$.

REMARK 1.4. ( $i$ ) In [8], it was shown that an analogous result to the one stated in Theorem 1.3 holds for a family of Riemannian exponential maps of the Lie group of orientation preserving $C^{\infty}$-diffeomorphisms of the circle $\mathbb{T}=\mathbb{R} / \mathbb{Z}$. In [16] we have improved this result showing that the exponential maps are Fréchet bianalytic diffeomorphisms near 0 .

(ii) While the proofs of Theorem 1.2 and Theorem 1.3 follow the same approach pioneered in [8], the integrals of the flow stemming from Noether's theorem are of no use in this higher dimensional situation. Instead, the proofs of these theorems are based on a novel interplay of the structure of the equations describing the geodesic flow from a Lagrangian perspective and the structure of the Euler equation.

Theorem 1.5. Assume that the Riemannian exponential map $\operatorname{Exp}_{k}$ for $k=0$ can be uniquely defined near 0 as a $C_{F}^{1}$ map in $C^{\infty}\left(\mathbb{T}^{2}, \mathbb{R}^{2}\right)$. Then, there is no neighborhood 
$V^{(0)}$ of 0 in $C^{\infty}\left(\mathbb{T}^{2}, \mathbb{R}^{2}\right)$ so that $\operatorname{Exp}_{0}$ is a $C_{F}^{1}$-diffeomorphism from $V^{(0)}$ onto a neighborhood of the identity map in $\mathcal{D}_{+}$.

The paper is organized as follows: Sections 2 and 3 are preliminary. Theorem 1.2 is proved in Section 4 and Theorem 1.3 in Section 5. To prove Theorem 1.3 we use a version of the inverse function theorem in a set-up with Fréchet spaces, discussed in Appendix A (Theorem A.5). The assumptions of Theorem A.5 are verified in Section 5. The key result, stated in Proposition 5.1, is that assumption (c) of Theorem A.5 holds. It says that for $k \geq 1$, a $\nu^{(k)}$-geodesic in $\mathcal{D}_{+}^{\ell}$ (with $\ell \geq 2 k+5$ ) issuing from the identity (with sufficiently small speed) and such that at $t=1$ it passes through an element in $\mathcal{D}_{+}^{\ell+1}$, actually evolves in $\mathcal{D}_{+}^{\ell+1}-$ a quite astonishing property for solutions of an evolution equation. Proposition 5.1 and Proposition 5.2, corresponding to assumption $(c)$ and, respectively, $(d)$ of Theorem A.5, are proved in Section 6. For the convenience of the reader, some elementary auxiliary results used to prove these propositions are collected in Appendix B. Theorem 1.5 is proved in Section 7.

We use standard notation. In particular, $H^{\ell}=H^{\ell}\left(\mathbb{T}^{2}, \mathbb{R}^{2}\right)$ denotes the space of $\mathbb{R}^{2}$-valued functions on $\mathbb{T}^{2}$ of Sobolev class $H^{\ell}$. Depending on the context, we will also use $H^{\ell}$ to denote the Sobolev space $H^{\ell}\left(\mathbb{T}^{2}, \mathbb{R}\right)$ or $H^{\ell}\left(\mathbb{T}^{2}\right.$, Mat $\left._{2 \times 2}\right)$ where Mat $2 \times 2$ denotes the linear space of $2 \times 2$ real valued matrices. Further, for $\ell \geq 3, \mathcal{D}_{+}^{\ell}=\mathcal{D}_{+}^{\ell}\left(\mathbb{T}^{2}\right)$ denotes the set of orientation preserving $C^{1}$-diffeomorphisms $\varphi: \mathbb{T}^{2} \rightarrow \mathbb{T}^{2}$ of class $H^{\ell}$. It is a Hilbert manifold modeled on $H^{\ell}$.

In the remainder of the paper, we will always identify a $C^{1}$-diffeomorphism $\varphi$ : $\mathbb{T}^{2} \rightarrow \mathbb{T}^{2}$ with a lift $\mathbb{R}^{2} \rightarrow \mathbb{R}^{2}$ of the form $T+f$ where $f: \mathbb{R}^{2} \rightarrow \mathbb{R}^{2}$ is periodic i.e., $f(x+\xi)=f(x)$ for any $x \in \mathbb{R}^{2}$ and $\xi \in \mathbb{Z}^{2}$, and $T \in S L(2 ; \mathbb{Z})$.

Acknowledgment. The first author would like to thank for the hospitality of the Mathematics Department of the Johns Hopkins University where part of this work has been done.

2. Group of orientation preserving diffeomorphisms. In this section we introduce some more notations and review some properties of spaces of maps from $\mathbb{T}^{2}$ onto itself - see [12] as well as [11], [22], [28], [29].

Throughout this section $s$ denotes an integer satisfying $s \geq 3$. Denote by $\mathcal{D}_{+}^{s}$ the set of all orientation preserving $C^{1}$-diffeomorphisms $\varphi: \mathbb{T}^{2} \rightarrow \mathbb{T}^{2}$ such that $\partial_{x_{j}} \varphi$ $(j=1,2)$ are in $H^{s-1}$ i.e.,

$$
\mathcal{D}_{+}^{s}:=\left\{\varphi: \mathbb{T}^{2} \rightarrow \mathbb{T}^{2} \mid C^{1}-\text { diffeomorphism; } \operatorname{det}(d \varphi)>0 ; d \varphi \in H^{s-1}\right\} .
$$

$\mathcal{D}_{+}^{s}$ is in a natural way a Hilbert manifold modeled on the Hilbert space $H^{s}=$ $H^{s}\left(\mathbb{T}^{2}, \mathbb{R}^{2}\right)$. An atlas of $\mathcal{D}_{+}^{s}$ can be described in terms of lifts of $\varphi$ in $\mathcal{D}_{+}^{s}$ i.e.,

$$
\mathbb{R}^{2} \rightarrow \mathbb{R}^{2}, x \mapsto T x+f(x) \text { where } f=\left(f_{1}, f_{2}\right) \in H^{s} \text { and } T \in S L(2 ; \mathbb{Z}) .
$$

For $j=1,2$, let $\mathrm{C}_{1}\left(f_{j}\right)$ and $\mathrm{C}_{2}\left(f_{j}\right)$ be short for the conditions on the function $f_{j}$, $\left|f_{j}(0)\right|<1 / 2$, respectively $0<f_{j}(0)<1$. Then the following set of Hilbert charts $(1 \leq i, j \leq 2, T \in S L(2 ; \mathbb{Z}))$ form an atlas of $\mathcal{D}_{+}^{s}$,

$$
\mathfrak{U}_{i, j, T}^{s}:=\left\{\varphi=T+f \mid f \in H^{s}, C_{i}\left(f_{1}\right), C_{j}\left(f_{2}\right), \operatorname{det}(T+d f)>0\right\} .
$$

Note that for $s \geq 3, H^{s}$ is a Banach algebra. Moreover generally, for any $s \geq 3$ and any $|m| \leq s$, the bilinear mapping

$$
H^{s} \times H^{m} \rightarrow H^{m},(f, g) \mapsto f g
$$


is bounded. (For $0 \leq m \leq 3$ this follows from the Sobolev embedding theorem and for $-3 \leq m \leq-1$ one argues by duality.) The fact that $H^{s}$ is an algebra can be used to show that for $s \geq 3$, the inverse $\varphi^{-1}$ of an element $\varphi$ in $\mathcal{D}_{+}^{s}$ is again in $\mathcal{D}_{+}^{s}, v \circ \varphi$ belongs to $H^{s}$ for any $v$ in $H^{s}$ and $\varphi$ in $\mathcal{D}_{+}^{s}$, and the right translation $R_{\varphi}: H^{s} \rightarrow H^{s}$ is a bounded linear map. The following results are well-known - see e.g. [11], [12].

Proposition 2.1. For any $s \geq 3$, the inverse and composition maps are continuous. More precisely, for any integer $m \geq 0$,

(i) $H^{s+m} \times \mathcal{D}_{+}^{s} \rightarrow H^{s}, \quad(v, \varphi) \mapsto v \circ \varphi$ is $C^{m}$-smooth;

(ii) $\mathcal{D}_{+}^{s+m} \rightarrow \mathcal{D}_{+}^{s}, \quad \varphi \mapsto \varphi^{-1}$ is $C^{m}$-smooth.

Remark 2.2. Note that by Proposition 2.1, the composition and the inverse maps, $\mathcal{D}_{+} \times \mathcal{D}_{+} \rightarrow \mathcal{D}_{+}$, respectively $\mathcal{D}_{+} \rightarrow \mathcal{D}_{+}$, are smooth, making $\mathcal{D}_{+}$into a Lie group. Its tangent space at the identity, $T_{\mathrm{id}} \mathcal{D}_{+}$, is the Lie algebra of $C^{\infty}$-smooth vector fields on $\mathbb{T}^{2}$.

3. The vector field $\mathcal{F}_{k}$. In this section we assume that $k$ and $\ell$ are integers satisfying $k \geq 1$ and $\ell \geq \ell_{k}:=2 k+5 .{ }^{1}$ For any $(\varphi, v)$ in $\mathcal{D}^{\ell} \times H^{\ell}$, consider

$$
\mathcal{F}_{k}(\varphi, v):=\left(v, F_{k}(\varphi, v)\right),
$$

where $F_{k}=R_{\varphi} \circ A_{k}^{-1} \circ B_{k}\left(v \circ \varphi^{-1}\right)$ is defined in (1.3). Note that each component of $B_{k}(u)$, defined in (1.5), is a polynomial of degree 2 in $u_{1}, u_{2}$, and its derivatives up to order $2 k$. Indeed, in the expression (1.5), the terms of order $2 k+1$ occurring in the first two terms on the r.h.s. of $(1.5)$ cancel out as $A_{k}((u \cdot \nabla) u)-(u \cdot \nabla) A_{k} u$ is in $H^{\ell-2 k}$, being the commutator $\left[A_{k},(u \cdot \nabla)\right]$ acting on $u$. Now since $A_{k}: H^{\ell} \rightarrow H^{\ell-2 k}$ as well as $R_{\varphi}: H^{j} \rightarrow H^{j}$ and its inverse $R_{\varphi}^{-1}$ (cf. Proposition 2.1) are bounded linear operators for $3 \leq j \leq \ell$, the composition $R_{\varphi} \circ A_{k} \circ R_{\varphi}^{-1}: H^{\ell} \rightarrow H^{\ell-2 k}$ is a bounded linear operator. Moreover, as $\ell \geq \ell_{k}$ and $H^{j}$ is a Banach algebra for $j \geq 3$, $B_{k}: H^{\ell} \rightarrow H^{\ell-2 k}$ and therefore $R_{\varphi} \circ B_{k} \circ R_{\varphi}^{-1}: H^{\ell} \rightarrow H^{\ell-2 k}$ are continuous maps. In particular

$$
\mathcal{A}_{k}: \mathcal{D}_{+}^{\ell} \times H^{\ell} \rightarrow \mathcal{D}_{+}^{\ell} \times H^{\ell-2 k}, \quad(\varphi, v) \mapsto\left(\varphi, R_{\varphi} \circ A_{k} \circ R_{\varphi}^{-1} v\right)
$$

and

$$
\mathcal{B}_{k}: \mathcal{D}_{+}^{\ell} \times H^{\ell} \rightarrow \mathcal{D}_{+}^{\ell} \times H^{\ell-2 k}, \quad(\varphi, v) \mapsto\left(\varphi, R_{\varphi} \circ B_{k} \circ R_{\varphi}^{-1} v\right) .
$$

are well-defined. Further let

$$
\mathcal{P r o j}_{2}: \mathcal{D}_{+}^{\ell} \times H^{\ell} \rightarrow H^{\ell},(\varphi, v) \mapsto v
$$

denote the projection onto the second component.

Proposition 3.1. Let $k \geq 1$, and $\ell \geq \ell_{k}=2 k+5$. Then

(i) the map $\mathcal{A}_{k}: \mathcal{D}_{+}^{\ell} \times H^{\ell} \rightarrow \mathcal{D}_{+}^{\ell} \times H^{\ell-2 k}$ defined in (3.1) is a $C^{1}$-diffeomorphism with inverse given by

$$
\mathcal{A}_{k}^{-1}: \mathcal{D}_{+}^{\ell} \times H^{\ell-2 k} \rightarrow \mathcal{D}_{+}^{\ell} \times H^{\ell}, \quad(\varphi, v) \mapsto\left(\varphi, R_{\varphi} \circ A_{k}^{-1} \circ R_{\varphi}^{-1} v\right) .
$$

(ii) The map $\mathcal{B}_{k}: \mathcal{D}_{+}^{\ell} \times H^{\ell} \rightarrow \mathcal{D}_{+}^{\ell} \times H^{\ell-2 k}$ defined by (3.2) is $C^{1}$-smooth.

\footnotetext{
${ }^{1}$ Some of the statements below remain valid for $\ell_{k}$ smaller.
} 
As a consequence, $F_{k}=\operatorname{Proj}_{2} \circ \mathcal{A}_{k}^{-1} \circ \mathcal{B}_{k}$ and

(iii) the vector field

$$
\mathcal{F}_{k}: \mathcal{D}_{+}^{\ell} \times H^{\ell} \rightarrow H^{\ell} \times H^{\ell},(\varphi, v) \mapsto\left(v, F_{k}(\varphi, v)\right)
$$

is $C^{1}$-smooth.

REMARK 3.2. The arguments used in [16] show that the vector field $\mathcal{F}_{k}$ described in Proposition 3.1 (iii) is in fact analytic.

To prove Proposition 3.1 we need two auxiliary lemmas. First, let us introduce the standard multi-index notation for differential operators in two independent variables i.e., for $n=\left(n_{1}, n_{2}\right)$ in $\mathbb{Z}_{\geq 0}^{2}$, we define $\partial_{x}^{n}$ to be the differential operator $\partial_{x_{1}}^{n_{1}} \partial_{x_{2}}^{n_{2}}$ of order $|n|=n_{1}+n_{2}$.

Lemma 3.3. Let $k \geq 1, \ell \geq \ell_{k}=2 k+5$, and $n=\left(n_{1}, n_{2}\right) \in \mathbb{Z}_{\geq 0}^{2}$. Then, for any $\varphi$ in $\mathcal{D}_{+}^{\ell}$ and $v$ in $H^{\ell}$, and for for any $1 \leq|n| \leq 2 k$,

$$
R_{\varphi} \circ \partial_{x}^{n} \circ R_{\varphi}^{-1} v=\sum_{1 \leq|j| \leq|n|} p_{n, j}(\varphi) \partial_{x}^{j} v
$$

where the coefficients $p_{n, j}(\varphi)$ are polynomials in $(\operatorname{det}(d \varphi))^{-1}$ and $\partial_{x}^{\alpha} \varphi_{m}(1 \leq|\alpha| \leq$ $|n|+1-|j|, m=1,2)$ with integer coefficients.

Proof. The proof of the claimed formula follows in a straightforward way using that the two components of $\left(\partial_{x}^{n} \varphi^{-1}\right) \circ \varphi$ are polynomials in $(\operatorname{det}(d \varphi))^{-1}$ and $\partial_{x}^{\alpha} \varphi_{m}$ $(1 \leq|\alpha| \leq|n|, m=1,2)$ with integer coefficients.

Lemma 3.4. For any integer $s \geq 3$, introduce the open subset $W^{s}:=\left\{f \in H^{s}\right.$ : $\left.f(x) \neq 0 \forall x \in \mathbb{T}^{2}\right\}$ of $H^{s}=H^{s}\left(\mathbb{T}^{2}, \mathbb{R}\right)$. Then, the map $W^{s} \rightarrow H^{s}, f \mapsto 1 / f$ is $C^{1}$-smooth.

Proof. Let $f$ in $W^{s}$, and $f+U_{\epsilon}$ be the neighborhood of $f$ in $H^{s}$ where

$$
U_{\epsilon}=\left\{g \in H^{s} \mid\|g\|_{H^{s}}<\epsilon\right\}
$$

and $\epsilon>0$ is so small that $\|g / f\|_{H^{s}}<1$. Such a choice is possible since $H^{s}$ is a Banach algebra for $s \geq 3$ and therefore $\|g / f\|_{H^{s}} \leq C\|g\|_{H^{s}}\|1 / f\|_{H^{s}}$ so that it suffices to pick $0<\epsilon<\left(C\|1 / f\|_{H^{s}}\right)^{-1}$. Then, $f+g$ is in $W^{s}$ for any $g \in U_{\epsilon}$, and $1 /(f+g)$ can be written in terms of a series

$$
\frac{1}{f+g}=\frac{1}{f}\left(1-\frac{g}{f}+\left(\frac{g}{f}\right)^{2}-\ldots\right)
$$

which converges uniformly in $U_{\epsilon}(f)$ to an element in $H^{s}$. In particular, the map $f+U_{\epsilon} \rightarrow H^{s}, f+g \mapsto 1 /(f+g)$ is $C^{1}$-smooth.

Corollary 3.5. For any $s \geq 4$, the map $\mathcal{D}_{+}^{s} \rightarrow H^{s-1}, \varphi \mapsto(\operatorname{det}(d \varphi))^{-1}$ is $C^{1}$-smooth.

Proof. The map $\mathcal{D}_{+}^{s} \rightarrow H^{s-1}, \varphi \mapsto(\operatorname{det}(d \varphi))^{-1}$, is the composition of the map $\mathcal{D}_{+}^{s} \rightarrow H^{s-1}, \varphi \mapsto \operatorname{det}(d \varphi)$, with the $C^{1}$-map $W^{s-1} \rightarrow H^{s-1}, f \mapsto 1 / f$. 
Proof of Proposition 3.1. (i) Clearly the map defined in (3.3) is the inverse of (3.1). In particular, this shows that $\mathcal{A}_{k}$ is bijective. By Lemma 3.3 and the definition of $A_{k}$ we have that

$$
\begin{aligned}
R_{\varphi} \circ A_{k} \circ R_{\varphi}^{-1} v & =v+\sum_{1 \leq i \leq k}(-1)^{i} R_{\varphi} \circ \Delta^{i} \circ R_{\varphi}^{-1} v \\
& =v+\sum_{1 \leq|j| \leq 2 k} q_{2 k, j}(\varphi) \partial_{x}^{j} v
\end{aligned}
$$

where the coefficients of the matrices $q_{2 k, j}(\varphi)$ are polynomials in $(\operatorname{det}(d \varphi))^{-1}$ and $\partial_{x}^{\alpha} \varphi_{m}(|\alpha| \leq 2 k+1-|j|, m=1,2)$ with integer coefficients. By Corollary 3.5 , the $\operatorname{map} \mathcal{D}_{+}^{\ell} \times \bar{H}^{\ell} \rightarrow\left(H^{\ell-2 k}\right)^{k^{*}}$

$$
(\varphi, v) \mapsto\left((\operatorname{det}(d \varphi))^{-1},\left(\left(\partial_{x}^{\alpha} \varphi_{1}\right),\left(\partial_{x}^{\alpha} \varphi_{2}\right)\right)_{1 \leq|\alpha| \leq 2 k},\left(\left(\partial_{x}^{\alpha} v_{1}\right),\left(\partial_{x}^{\alpha} v_{2}\right)\right)_{0 \leq|\alpha| \leq 2 k}\right)
$$

is a $C^{1}$-map where $k^{*}=4 k(2 k+3)-1$. Since for $\ell \geq \ell_{k}=2 k+5, H^{\ell-2 k}$ is a Banach algebra, we conclude that the r.h.s. of (3.5) is $C^{1}$-smooth and hence that $\mathcal{A}_{k}$ is $C^{1}$-smooth. Moreover, for any $\left(\varphi_{0}, v_{0}\right)$ in $\mathcal{D}_{+}^{\ell} \times H^{\ell}$, the differential $d_{\left(\varphi_{0}, v_{0}\right)} \mathcal{A}_{k}$ : $H^{\ell} \times H^{\ell} \rightarrow H^{\ell} \times H^{\ell-2 k}$ is of the form

$$
d_{\left(\varphi_{0}, v_{0}\right)} \mathcal{A}_{k}(\delta \varphi, \delta v)=\left(\begin{array}{cc}
\delta \varphi & 0 \\
\Lambda(\delta \varphi) & R_{\varphi_{0}} \circ A_{k} \circ R_{\varphi_{0}}^{-1} \delta v
\end{array}\right)
$$

where

$$
\Lambda: H^{\ell} \rightarrow H^{\ell-2 k}, \quad \text { and } \quad R_{\varphi_{0}} \circ A_{k} \circ R_{\varphi_{0}}^{-1}: H^{\ell} \rightarrow H^{\ell-2 k}
$$

are bounded linear maps. As the latter map has a bounded inverse, $d_{\left(\varphi_{0}, v_{0}\right)} \mathcal{A}_{k}$ is a linear isomorphism and, by the inverse function theorem, $\mathcal{A}_{k}$ is a local $C^{1}$ diffeomorphism. Since we have seen that $\mathcal{A}_{k}$ is bijective, assertion $(i)$ then follows. The proof of item $(i i)$ is similar to the proof of the $C^{1}$-smoothness of $\mathcal{A}_{k}$ in part $(i)$.

Proposition 3.1 allows to apply the existence and uniqueness theorems of ODE's in Banach spaces (see e.g. [22]) to the initial value problems (1.2) on $\mathcal{D}_{+}^{\ell} \times H^{\ell}$ with $\ell \geq \ell_{k}=2 k+5$. As for any $k \geq 1$, the element $(\mathrm{id}, 0)$ in $\mathcal{D}_{+}^{\ell} \times T_{\mathrm{id}} \mathcal{D}_{+}$is a stationary solution of (1.2) one gets the following result.

TheOREM 3.6. Let $k \geq 1$ and $\ell \geq \ell_{k}=2 k+5$. Then there exists an open neighborhood $V_{k ; \ell}$ of 0 in $H^{\ell}$ so that, for any $v_{0}$ in $V_{k ; \ell}$, the initial value problem (1.2) has a unique $C^{1}$-solution

$$
(-2,2) \rightarrow \mathcal{D}_{+}^{\ell} \times H^{\ell}, \quad t \mapsto(\varphi(t), v(t)) .
$$

Moreover, the flow map is $C^{1}$,

$$
(-2,2) \times V_{k ; \ell} \rightarrow \mathcal{D}_{+}^{\ell} \times H^{\ell}, \quad\left(t, v_{0}\right) \mapsto\left(\varphi\left(t ; v_{0}\right), v\left(t ; v_{0}\right)\right)
$$

REMARK 3.7. Note that Theorem 3.6 does not exclude that $\bigcap_{\ell \geq \ell_{k}} V_{k ; \ell}=\{0\}$. This possibility is ruled out by Theorem 4.1 of the next section.

As discussed in the introduction, the initial value problem (1.2) on $\mathcal{D}_{+}^{\ell} \times H^{\ell}$ is closely related to the initial value problems (1.6)-(1.7) on $\mathcal{D}_{+}^{\ell}$ and $H^{\ell}$ respectively. A 
solution $(\varphi, v)$ in $C^{1}\left((-2,2), \mathcal{D}_{+}^{\ell} \times H^{\ell}\right)$ of (1.2) corresponds via (1.6) to a solution of (1.7) in $C^{0}\left((-2,2), H^{\ell}\right) \cap C^{1}\left((-2,2), H^{\ell-1}\right)$. To check this, note that from the first equation of (1.2) and (1.6) we have that

$$
u=v \circ \varphi^{-1}
$$

so that $u(t)$ is in $H^{\ell}$ for any $-2<t<2$. Further, $t \mapsto \varphi(t)$ evolves in $\mathcal{D}_{+}^{\ell}$ and hence so does $t \mapsto \varphi^{-1}(t)$; they are continuous within the same time interval. Thus, by Proposition 2.1,

$$
(-2,2) \rightarrow H^{\ell}, \quad t \mapsto u(t)=v(t) \circ \varphi^{-1}(t)
$$

is a continuous curve in $H^{\ell}$ and a $C^{1}$-curve in $H^{\ell-1}$, emanating from $v(0) \circ \varphi^{-1}(0)=$ $v_{0}$. Altogether, we have

$$
u \in C^{0}\left((-2,2), H^{\ell}\right) \cap C^{1}\left((-2,2), H^{\ell-1}\right) .
$$

A direct computation shows that $u$ is a solution of $(1.7)$ on $(-2,2) \times \mathbb{T}^{2}$.

4. Riemannian exponential maps. Throughout this section we assume that $k$ and $\ell$ are integers satisfying $k \geq 1$, and $\ell \geq \ell_{k}:=2 k+5$. By Theorem 3.6, there exists a neighborhood $V_{\ell_{k}}^{(k)}:=V_{k ; \ell_{k}}$ of 0 in $H^{\ell_{k}}$ so that for any $v_{0}$ in $V_{\ell_{k}}^{(k)}$, the initial value problem (1.2) has a unique $C^{1}$-solution

$$
(-2,2) \rightarrow \mathcal{D}_{+}^{\ell_{k}} \times H^{\ell_{k}}, \quad t \mapsto(\varphi(t), v(t))
$$

We emphasize the dependence on the initial data by writing $t \mapsto\left(\varphi\left(t ; v_{0}\right), v\left(t ; v_{0}\right)\right)$ instead of $t \mapsto(\varphi(t), v(t))$. Moreover, the map

$$
(-2,2) \times V_{\ell_{k}}^{(k)} \rightarrow \mathcal{D}_{+}^{\ell_{k}} \times H^{\ell_{k}},\left(t, v_{0}\right) \mapsto\left(\varphi\left(t ; v_{0}\right), v\left(t ; v_{0}\right)\right)
$$

is $C^{1}$. Hence, in particular, the exponential map

$$
\operatorname{Exp}_{k, \ell_{k}}: V_{\ell_{k}}^{(k)} \rightarrow \mathcal{D}_{+}^{\ell_{k}}, \quad v_{0} \mapsto \varphi\left(1 ; v_{0}\right)
$$

is $C^{1}$. In this section we want to study the restriction of $\operatorname{Exp}_{k, \ell_{k}}$ to $V_{\ell_{k}}^{(k)} \cap C^{\infty}\left(\mathbb{T}^{2}, \mathbb{R}^{2}\right)$. As explained at the end of the previous section, for any $v_{0}$ in $V_{\ell_{k}}^{(k)}$, the curve $t \mapsto u(t)=$ $v(t) \circ \varphi(t)^{-1}$ is a solution of equation (1.7) in $C^{0}\left((-2,2), H^{\ell_{k}}\right) \cap C^{1}\left((-2,2), H^{\ell_{k}-1}\right)$.

We start by deriving transport equations for $\left(A_{k} u\right) \circ \varphi$ and $d \varphi$. This will be helpful to study the regularity properties of the exponential map as we will see shortly. As $C^{1}\left((-2,2), H^{3}\right) \hookrightarrow C^{1}\left((-2,2) \times \mathbb{T}^{2}\right)$ in view of the Sobolev embedding theorem, one obtains by pointwise differentiation

$$
\left[\left(A_{k} u\right) \circ \varphi\right]^{\cdot}=\left(A_{k} \dot{u}\right) \circ \varphi+\left[\left(d A_{k} u\right) \circ \varphi\right] \dot{\varphi}
$$

Notice that the latter identity actually holds in $H^{\ell_{k}-2 k-1}$. As $\dot{\varphi}=v=u \circ \varphi$, (1.7) leads to the following linear initial value problem in $H^{\ell_{k}-2 k-1}$ for $w=w(t):=$ $\left(A_{k} u(t)\right) \circ \varphi(t)$

$$
\left\{\begin{array}{l}
\dot{w}+\left[((\operatorname{div} u) \circ \varphi) \operatorname{Id}+(d u)^{\dagger} \circ \varphi\right] w=0 \\
w(0)=A_{k} v_{0}
\end{array}\right.
$$


On the other hand, differentiating equation (1.6), considered in $H^{\ell_{k}}$, with respect to the spatial variables one gets

$$
\left\{\begin{array}{l}
(d \varphi)^{\cdot}=[(d u) \circ \varphi] d \varphi \\
d \varphi(0)=\mathrm{Id}
\end{array}\right.
$$

which we view as a linear system of ODE's in $H^{\ell_{k}-1}$ for the two columns of the Jacobian $d \varphi$. We will use (4.2) and (4.3) to prove that any solution curve $t \mapsto$ $\left(\varphi\left(t ; v_{0}\right), v\left(t ; v_{0}\right)\right)$ in $C^{1}\left((-2,2), \mathcal{D}_{+}^{\ell_{k}} \times H^{\ell_{k}}\right)$ emanating from $(\varphi(0), v(0))=\left(\mathrm{id}, v_{0}\right)$, with $v_{0}$ in $V_{\ell_{k}}^{(k)} \cap H^{\ell}$ and $\ell \geq \ell_{k}$, actually evolves in $\mathcal{D}_{+}^{\ell} \times H^{\ell}$.

Theorem 4.1. Let $k \geq 1$ and $\ell \geq \ell_{k}=2 k+5$. If $v_{0}$ is in $V_{\ell}^{(k)}:=V_{\ell_{k}}^{(k)} \cap H^{\ell}$, then there exists a unique solution $(\varphi, v)$ of $(1.2)$ in $C^{1}\left((-2,2), \mathcal{D}_{+}^{\ell} \times H^{\ell}\right)$. Moreover, $u:=v \circ \varphi^{-1}$ is a solution of $(1.7)$ in $C^{0}\left((-2,2), H^{\ell}\right) \cap C^{1}\left((-2,2), H^{\ell-1}\right)$.

REMARK 4.2. The solution $(\varphi, v)$ in Theorem 4.1 depends $C^{1}$-smoothly on the initial data $v_{0} \in V_{\ell}^{(k)}$ so that $(\varphi, v) \in C^{1}\left((-2,2) \times V_{\ell}^{(k)}, \mathcal{D}_{+}^{\ell} \times H^{\ell}\right)$ (cf. e.g. [22], Chapter IV).

Proof of Theorem 4.1. We argue by induction with respect to $\ell$. For $\ell=\ell_{k}$, the statement follows from the definition of $V_{\ell_{k}}^{(k)}$, and the discussion at the end of Section 3. Now, assume that the proposition holds for a fixed $\ell \geq \ell_{k}$ i.e., assume that any given $v_{0}$ in $V_{\ell}^{(k)}$, the solution curve $(\varphi, v)$ of $(1.2)$ is in $C^{1}\left((-2,2), \mathcal{D}_{+}^{\ell} \times H^{\ell}\right)$ and the corresponding solution $u$ of $(1.7)$ lies in $C^{0}\left((-2,2), H^{\ell}\right) \cap C^{1}\left((-2,2), H^{\ell-1}\right)$. Let $v_{0} \in V_{\ell+1}^{(k)}$. As $V_{\ell+1}^{(k)} \subseteq V_{\ell}^{(k)}$ we get from the induction hypothesis that $w=\left(A_{k} u\right) \circ \varphi$ belongs to $C^{0}\left((-2,2), H^{\ell-2 k}\right)$ - see Propositon 2.1 (i). Moreover for any $1 \leq j \leq 2 k$

$$
\left[\left(\operatorname{div} u \cdot \operatorname{Id}+(d u)^{\dagger}\right) \circ \varphi\right] \in C^{0}\left((-2,2), \mathcal{L}\left(H^{\ell-j}, H^{\ell-j}\right)\right)
$$

where $\mathcal{L}\left(H^{s}, H^{s}\right)$ denotes the space of bounded linear operators from $H^{s}$ to $H^{s}$. At this point it is crucial that we assume $k \geq 1$. It garantees that $2 k-1 \geq 1$ so that we can apply (4.4) for $j=2 k-1$ to conclude that (4.2) is a linear ODE in $H^{\ell+1-2 k}$. As $w(0)=A_{k} v_{0} \in H^{\ell+1-2 k}$, it then follows that $w$ is in $C^{1}\left((-2,2), H^{\ell+1-2 k}\right)$. On the other hand, since $\varphi^{-1}$ is in $C^{0}\left((-2,2), H^{\ell}\right)$ (cf. Proposition 2.1 (ii)), $w \circ \varphi^{-1}=A_{k} u$ is in $C^{0}\left((-2,2), H^{\ell+1-2 k}\right)$ i.e., $u$ belongs to $C^{0}\left((-2,2), H^{\ell+1}\right)$. We now want to show that $u$ lies in $C^{1}\left((-2,2), H^{\ell}\right)$. Recall that by the induction hypothesis, (1.7) holds in $H^{\ell-2 k-1}$. Hence when integrating (1.7) with respect to $t$ in $H^{\ell-2 k-1}$, we get

$$
A_{k} u(t)=v_{0}-\int_{0}^{t}\left(\left(d A_{k} u\right) u+\left(\operatorname{div} u \cdot \operatorname{Id}+(d u)^{\dagger}\right) A_{k} u\right) d \tau
$$

As by the considerations above, the integrand in the latter formula is in $C^{0}\left((-2,2), H^{\ell-2 k}\right)$, it follows that $A_{k} u \in C^{1}\left((-2,2), H^{\ell-2 k}\right)$. Altogether we have

$$
u \in C^{0}\left((-2,2), H^{\ell+1}\right) \cap C^{1}\left((-2,2), H^{\ell}\right) .
$$

Furthermore, as $\varphi$ is in $C^{1}\left((-2,2), \mathcal{D}_{+}^{\ell}\right)$, and $u$ is in $C^{0}\left((-2,2), H^{\ell+1}\right),(d u) \circ \varphi$ is in $C^{0}\left((-2,2), \mathcal{L}\left(H^{\ell}, H^{\ell}\right)\right)$ so that $(4.3)$ is a linear system of ODEs in $H^{\ell}$ for the two columns of the Jacobian $d \varphi$. Integrating (4.3) with respect to $t$ in $H^{\ell}$, one argues as above to conclude that the columns of $d \varphi$ are in $C^{1}\left((-2,2), H^{\ell}\right)$, and thus that $\varphi$ is in $C^{1}\left((-2,2), \mathcal{D}_{+}^{\ell+1}\right)$. Moreover, $v=u \circ \varphi$ is in $C^{0}\left((-2,2), H^{\ell+1}\right)$. By $(1.2)$, 
$\dot{v}=F_{k}(\varphi, v)$. By Proposition 3.1, $\dot{v}$ is in $C^{0}\left((-2,2), H^{\ell+1}\right)$. Arguing as above one then concludes that $(\varphi, v)$ is in $C^{1}\left((-2,2), \mathcal{D}_{+}^{\ell+1} \times H^{\ell+1}\right)$.

Proof of Theorem 1.2. Theorem 1.2 is an immediate consequence of Theorem 4.1 with $V^{(k)}$ given by $V^{(k)}:=V_{\ell_{k}}^{(k)} \cap C^{\infty}\left(\mathbb{T}^{2}, \mathbb{R}^{2}\right)$.

By shrinking $V_{\ell_{k}}^{(k)}$ if necessary, we can assume that the image of (4.1) is contained in the Hilbert chart

$$
\mathfrak{U}_{1,1, \text { id }}^{\ell_{k}}:=\left\{\mathbf{i d}+g\left|g \in H^{\ell},\right| g_{i}(0) \mid<1 / 2(i=1,2), \operatorname{det}(\operatorname{Id}+d g)>0\right\}
$$

defined in (2.1b). Note that $d_{0} \operatorname{Exp}_{k, \ell_{k}}=\operatorname{Id}_{H^{\ell_{k}}}$. It then follows from the inverse function theorem that by shrinking $V_{\ell_{k}}^{(k)}$ further, if necessary, one can ensure that there exists an open neighborhood $U_{\ell_{k}}^{(k)}$ of id in $\mathcal{D}_{+}^{\ell_{k}}$ such that

$$
\operatorname{Exp}_{k, \ell_{k}}: V_{\ell_{k}}^{(k)} \rightarrow U_{\ell_{k}}^{(k)}
$$

is a $C^{1}$-diffeomorphism and $U_{\ell_{k}}^{(k)} \subseteq \mathfrak{U}_{1,1, \text { id }}^{\ell_{k}}$. In what follows we will assume that the latter two conditions are satisfied.

Theorem 4.1 allows to define the exponential map on a neighborhood of 0 in $T_{\mathrm{id}} \mathcal{D}_{+}^{\ell}$ for any $\ell \geq \ell_{k}$. Recall that for any $k \geq 1$ and $\ell \geq \ell_{k}, V_{\ell}^{(k)}=V_{\ell_{k}}^{(k)} \cap H^{\ell}$, and

$$
V^{(k)}=V_{\ell_{k}}^{(k)} \cap C^{\infty}\left(\mathbb{T}^{2}, \mathbb{R}^{2}\right)=\bigcap_{\ell \geq \ell_{k}} V_{\ell}^{(k)} .
$$

Define for any $\ell \geq \ell_{k}$,

$$
\operatorname{Exp}_{k, \ell}: V_{\ell}^{(k)} \rightarrow \mathcal{D}_{+}^{\ell}, \quad v_{0} \mapsto \varphi\left(1 ; v_{0}\right)
$$

By Theorem 4.1, the restriction $\operatorname{Exp}_{k, \ell}$ of $\operatorname{Exp}_{k, \ell_{k}}$ to $V_{\ell}^{(k)}$ takes values in $\mathcal{D}_{+}^{\ell}$ and by Remark 4.2, $\operatorname{Exp}_{k, \ell}: V_{\ell}^{(k)} \rightarrow \mathcal{D}_{+}^{\ell}$ is a $C^{1}$-map. But then, the restriction $\operatorname{Exp}_{k}$ of $\operatorname{Exp}_{k, \ell_{k}}$ to $V^{(k)}$ takes values in $\mathcal{D}_{+}$,

$$
\operatorname{Exp}_{k}: V^{(k)} \rightarrow \mathcal{D}_{+}
$$

5. $C_{F}^{1}$-smooth charts of id in $\mathcal{D}_{+}$. Theorem 1.3 states that, for any integer $k \geq 1$, the exponential map

$$
\left.\operatorname{Exp}_{k}\right|_{V^{(k)}}: V^{(k)} \rightarrow \mathcal{D}_{+}
$$

can be used to define a $C_{F}^{1}$-smooth chart of the identity in $\mathcal{D}_{+}$. To prove Theorem 1.3 we will use the following two propositions which we state in a slightly stronger version than needed. Denote $U_{\ell}^{(k)}:=U_{\ell_{k}}^{(k)} \cap H^{\ell}$.

Proposition 5.1. For any $k \geq 1$ and any $\ell \geq \ell_{k}:=2 k+5, \operatorname{Exp}_{k, \ell}$ maps $V_{\ell}^{(k)}$ onto $U_{\ell}^{(k)}$.

Proposition 5.2. Let $k \geq 1, \ell \geq \ell_{k}=2 k+5$, and assume that $v_{0}$ belongs to $V_{\ell+2}^{(k)}$. Then

$$
\left(d_{v_{0}} \operatorname{Exp}_{k, \ell}\right)\left(H^{\ell} \backslash H^{\ell+1}\right) \subseteq H^{\ell} \backslash H^{\ell+1}
$$


We will prove these two propositions in the next section.

Proof of Theorem 1.3. We want to apply the inverse function theorem in Fréchet spaces as stated in Theorem A.5 of Appendix A. Fix $k \geq 1$ and let $\ell_{k}=2 k+5$. To match the notation of this theorem, we write $\ell=\ell_{k}+n, n \geq 0$ and define for any integer $n \geq 0$

$$
X_{n}:=H^{\ell}, \quad Y_{n}:=H^{\ell}, \quad \text { and } \quad V_{n}:=V_{\ell}^{(k)}, \quad U_{n}:=\operatorname{Exp}_{k, \ell}\left(V_{\ell}^{(k)}\right)
$$

where $V_{\ell}^{(k)}$ and $\operatorname{Exp}_{k, \ell}$ were introduced in Section 4. Further, define the map $f$ of Theorem A.5 by $f:=\operatorname{Exp}_{k, \ell_{k}}: V_{0} \rightarrow U_{0}$. By the choice of $V_{\ell_{k}}^{(k)}$, for any $n \geq 0, U_{n}$ is contained in $\mathfrak{U}_{1,1}^{\ell}$ and therefore can be identified with an open neighborhood of 0 in $X_{n}=H^{\ell}$. As by construction $\operatorname{Exp}_{k, \ell_{k}}: V_{\ell_{k}}^{(k)} \rightarrow U_{\ell_{k}}^{(k)}$ is a $C^{1}$-diffeomorphism, item (a) of Theorem A.5 is verified. Assumption (b) of the latter theorem holds in view of Theorem 4.1 and Remark 4.2, whereas items $(c)$ and $(d)$ hold by Proposition 5.1, and Proposition 5.2, respectively. Finally, upon setting $\tilde{V}^{(k)}=V^{(k)}:=\cap_{n \geq 0} V_{n}$ and $U^{(k)}:=\cap_{n \geq 0} U_{n}$, Theorem 1.3 then follows from Theorem A.5.

6. Proof of Propositions 5.1 and 5.2. In this section we prove Propositions 5.1 and 5.2 which were used in the proof of Theorem 1.3. Assume that $\ell \geq 2 k+5$ and let $\varphi \in C^{1}\left((-2,2), \mathcal{D}_{+}^{\ell}\right)$ and $u \in C^{0}\left((-2,2), H^{\ell}\right) \cap C^{1}\left((-2,2), H^{\ell-1}\right)$ be solutions of (1.6) and (1.7) respectively. For any given $x$ in $\mathbb{T}^{2}$ and $-2<t<2$ consider the linear system of ODE's

$$
\left\{\begin{array}{l}
\dot{\Phi}-[(d u) \circ \varphi] \Phi=0 \\
\left.\Phi\right|_{t=0}=\mathrm{Id}
\end{array}\right.
$$

Note that by the Sobolev embedding theorem for any given $x \in \mathbb{T}^{2}$ the elements of the $2 \times 2$ matrix $(d u) \circ \varphi$ are continuous real-valued functions of $t$ on $(-2,2)$. Denote by $\Phi^{t} \equiv \Phi^{t}(x)$ the fundamental matrix of (6.1). Arguing as in $\S 4$ one sees that

$$
\Phi^{t}(x)=d_{x} \varphi(t) \quad \forall x \in \mathbb{T}^{2}, \quad \text { and } \quad \forall t \in(-2,2) .
$$

Further, for any $x$ in $\mathbb{T}^{2}$ and $-2<t<2$ denote by $\Psi^{t}=\Psi^{t}(x)$ the $2 \times 2$ fundamental matrix of the linear system

$$
\left\{\begin{array}{l}
\dot{\Psi}+\left[\left(\operatorname{div} u \cdot \operatorname{Id}+(d u)^{\dagger}\right) \circ \varphi\right] \Psi=0 \\
\left.\Psi\right|_{t=0}=\operatorname{Id}
\end{array}\right.
$$

Regarding (6.3) as a linear ODE for $\Psi$ in $H^{\ell-1}$ one concludes that $\Psi \in$ $C^{1}\left((-2,2), H^{\ell-1}\right)$. Moreover, the arguments in $\S 4$ show that

$$
\left(A_{k} u\right) \circ \varphi=\Psi^{t} A_{k} u(0) .
$$

Proof of Proposition 5.1. We argue by induction. For $\ell=\ell_{k}, \operatorname{Exp}_{k, \ell_{k}}: V_{\ell_{k}}^{(k)} \rightarrow$ $U_{\ell_{k}}^{(k)}$ is a $C^{1}$-diffeomorphism by the definition of $V_{\ell_{k}}^{(k)}$ and $U_{\ell_{k}}^{(k)}$. Now, given an arbitrary $\ell \geq \ell_{k}$, assume that any solution $(\varphi, v)$ of $(1.2)$ with $v_{0} \in V_{\ell_{k}}^{(k)}$ for which $\varphi(1)$ is in $U_{\ell}^{(k)}$, has initial data (id, $\left.v_{0}\right)$ with $v_{0}$ in $V_{\ell}^{(k)}$. By Theorem 4.1 , it is equivalent to assume that for the given data, the solution curve $(\varphi, v)$ be in $C^{1}\left((-2,2), \mathcal{D}_{+}^{\ell} \times H^{\ell}\right)$. Then, $u=v \circ \varphi^{-1}$ is a solution of (1.7) in $C^{0}\left((-2,2), H^{\ell}\right) \cap C^{1}\left((-2,2), H^{\ell-1}\right)$. We 
need to show that, if in addition $\operatorname{Exp}_{k, \ell}\left(v_{0}\right)=\varphi(1)$ is in $\mathcal{D}_{+}^{\ell+1}$, then $v_{0}$ must be in $H^{\ell+1}$, and hence that $(\varphi, v)$ is in $C^{1}\left((-2,2), \mathcal{D}_{+}^{\ell+1} \times H^{\ell+1}\right)$. To this end note that

$$
-\Delta(u \circ \varphi)=[(d u) \circ \varphi](-\Delta \varphi)-\sum_{1 \leq i, j \leq 2}\left[(d \varphi)(d \varphi)^{\dagger}\right]_{i j}\left[\left(\partial_{x_{i}} \partial_{x_{j}} u\right) \circ \varphi\right] .
$$

Hence, applying $-\Delta$ to the identity $\dot{\varphi}=u \circ \varphi$ yields

$$
(-\Delta \varphi)^{\cdot}-[(d u) \circ \varphi](-\Delta \varphi)=\left(\mathcal{P}_{\lambda} u\right) \circ \varphi-\lambda u \circ \varphi
$$

where $\mathcal{P}_{\lambda}$ is the second order elliptic differential operator

$$
\mathcal{P}_{\lambda}=\lambda-\sum_{1 \leq i, j \leq 2} p_{i j} \partial_{x_{i}} \partial_{x_{j}}
$$

where the coefficients $p_{i j}=p_{i j}(t, x), 1 \leq i, j \leq 2$,

$$
p_{i j}:=\left[\left\{(d \varphi)(d \varphi)^{\dagger}\right\} \circ \varphi^{-1}\right]_{i j}
$$

are in $C^{0}\left((-2,2), H^{\ell-1}\right)$ (cf. Proposition 2.1). We will choose the parameter $\lambda \geq 1$ so that inequality (B.2) in Lemma B.2 in Appendix B holds uniformly in $0 \leq t \leq 1$. Our strategy is to find a formula relating $\varphi(1)$ and $v_{0}$ which will allow us to show that $v_{0}$ actually lies in $H^{\ell+1}$. To this end it turns out to be more convenient not to work with identity (6.6) but rather the one which arises by applying $-\Delta$ to (6.6) once more i.e.,

$$
\left\{\begin{array}{l}
\left(\Delta^{2} \varphi\right)^{\cdot}-[(d u) \circ \varphi]\left(\Delta^{2} \varphi\right)=\left(\mathcal{P}_{\lambda}^{\dagger} \mathcal{P}_{\lambda} u\right) \circ \varphi+g \\
\Delta^{2} \varphi(0)=0
\end{array}\right.
$$

where $g$ is in $C^{0}\left((-2,2), H^{\ell-3}\right)$. Here we have used the fact that $\left(\mathcal{P}_{\lambda} \mathcal{P}_{\lambda} u\right) \circ \varphi=$ $\left(\mathcal{P}_{\lambda}^{\dagger} \mathcal{P}_{\lambda} u\right) \circ \varphi+\ldots$, where $\ldots$ stand for terms in $C^{0}\left((-2,2), H^{\ell-3}\right)$ which are included in $g$. By the Sobolev embedding theorem $C^{1}\left((-2,2), H^{\ell-4}\right) \hookrightarrow C^{1}\left((-2,2) \times \mathbb{T}^{2}, \mathbb{R}^{2}\right)$ and hence for any fixed $x \in \mathbb{T}^{2}$ we can view (6.8) as an inhomogeneous linear ODE for $\Delta^{2} \varphi$. This implies that for any given $x \in \mathbb{T}^{2}, \Delta^{2} \varphi$ admits a representation of the form

$$
\begin{aligned}
\Delta^{2} \varphi(t) & =\Phi^{t} \int_{0}^{t}\left(\Phi^{s}\right)^{-1} \circ R_{\varphi(s)}\left[\mathcal{P}_{\lambda}^{\dagger}(s) \mathcal{P}_{\lambda}(s) u(s)\right] d s \\
& +\Phi^{t} \int_{0}^{t}\left(\Phi^{s}\right)^{-1} g d s
\end{aligned}
$$

where $\Phi^{t}=\Phi^{t}(x)=d_{x} \varphi(t)$ is the fundamental solution of (6.1). As $t \mapsto \Phi^{t}$ is in $C^{1}\left((-2,2), H^{\ell-1}\right)$ and $g \in C^{0}\left((-2,2), H^{\ell-3}\right)$, the integral in $(6.9)$ is in $H^{\ell-3}$ and therefore represents an element in $H^{\ell-3}$. Hence

$$
\Delta^{2} \varphi(t)=\Phi^{t} \int_{0}^{t}\left(\Phi^{s}\right)^{-1} \circ R_{\varphi(s)}\left[\mathcal{P}_{\lambda}^{\dagger}(s) \mathcal{P}_{\lambda}(s) u(s)\right] d s+\ldots
$$

where $\ldots$ stand for terms in $C^{1}\left((-2,2), H^{\ell-3}\right)$. Note that the integral in $(6.10)$ converges in $H^{\ell-4}$. In particular, $(6.10)$ can be considered not only as a pointwise equality but also as an equality in $H^{\ell-4}$. 
To analyze (6.10) further, recall that for any given $-2<t<2$ and $x \in \mathbb{T}^{2}$,

$$
\left(A_{k} u(t)\right) \circ \varphi(t)=\Psi^{t} A_{k} v_{0}
$$

where $\Psi^{t}$ is the fundamental matrix of (6.3). Hence, for any $-2<t<2$, the solution of (1.7) reads

$$
u(t)=A_{k}^{-1} \circ R_{\varphi(t)}^{-1} \circ \Psi^{t}\left(A_{k} v_{0}\right)
$$

Upon substituting the latter into the r.h.s. of equation (6.10), we get that $\Delta^{2} \varphi(t)$ is equal to

$$
\begin{aligned}
& \Phi^{t} \int_{0}^{t}\left(\Phi^{s}\right)^{-1} \circ R_{\varphi(s)}\left[\mathcal{P}_{\lambda}^{\dagger}(s) \mathcal{P}_{\lambda}(s)\left\{A_{k}^{-1} \circ R_{\varphi(s)}^{-1} \circ \Psi^{s}\left(A_{k} v_{0}\right)\right\}\right] d s+\ldots \\
= & \Phi^{t} \int_{0}^{t}\left(\Phi^{s}\right)^{-1} \circ R_{\varphi(s)} \circ A_{k}^{-1 / 2} \circ \mathcal{P}_{\lambda}^{\dagger}(s) \circ \mathcal{P}_{\lambda}(s) \circ A_{k}^{-1 / 2} \circ R_{\varphi(s)}^{-1} \circ \Psi^{s}\left(A_{k} v_{0}\right) d s \\
+ & \Phi^{t} \int_{0}^{t}\left(\Phi^{s}\right)^{-1} \circ R_{\varphi(s)} \circ A_{k}^{-1 / 2} \circ\left[A_{k}^{1 / 2}, \mathcal{P}_{\lambda}^{\dagger}(s) \mathcal{P}_{\lambda}(s)\right] \circ A_{k}^{-1} \circ R_{\varphi(s)}^{-1} \circ \Psi^{s}\left(A_{k} v_{0}\right) d s \\
+ & \ldots
\end{aligned}
$$

where $\ldots$ again stand for terms in $C^{0}\left((-2,2), H^{\ell-3}\right)$, and $\left[A_{k}^{1 / 2}, \mathcal{P}_{\lambda}^{\dagger} \mathcal{P}_{\lambda}\right]$ denotes the commutator

$$
\left[A_{k}^{1 / 2}, \mathcal{P}_{\lambda}^{\dagger} \mathcal{P}_{\lambda}\right]=A_{k}^{1 / 2} \circ \mathcal{P}_{\lambda}^{\dagger} \mathcal{P}_{\lambda}-\mathcal{P}_{\lambda}^{\dagger} \mathcal{P}_{\lambda} \circ A_{k}^{1 / 2}
$$

Note that, by the induction hypothesis, the coefficients of the differential operator $\mathcal{P}_{\lambda}^{\dagger} \mathcal{P}_{\lambda}$ are in $C^{1}\left((-2,2), H^{\ell-3}\right)$. By Remark B.5 at the end of Appendix B below, the second term on the r.h.s. of $(6.11)$ is in $C^{0}\left((-2,2), H^{\ell-3}\right)$. Hence, at $t=1$, we have

$$
\Delta^{2} \varphi(1)=\Phi^{1} \mathcal{Q}_{k}\left(A_{k} v_{0}\right)+\ldots
$$

where ... stand for elements in $H^{\ell-3}$, and where for $f \in H^{\ell-2 k}, \mathcal{Q}_{k}$ is defined by

$$
\mathcal{Q}_{k} f:=\int_{0}^{1}\left(\Phi^{s}\right)^{-1} \circ R_{\varphi(s)} \circ A_{k}^{-1 / 2} \circ \mathcal{P}_{\lambda}^{\dagger}(s) \circ \mathcal{P}_{\lambda}(s) \circ A_{k}^{-1 / 2} \circ R_{\varphi(s)}^{-1} \circ \Psi^{s} f d s .
$$

Here the integration is carried out in $H^{\ell-4}$. Recall that $\lambda$ is chosen so that (B.2) in Lemma B.2 holds. We claim that $\mathcal{Q}_{k}$ extends to a bounded linear operator $\mathcal{Q}_{k}$ : $H^{-(k-2)} \rightarrow H^{k-2}$. First note that for any $0 \leq j \leq \ell-1$, the linear mapping $R_{\varphi(s)}: H^{j} \rightarrow H^{j}$ is bounded uniformly in $0 \leq s \leq 1$. Moreover, for every $0 \leq s \leq 1$, $R_{\varphi(s)}$ is well defined on the spaces of distributions $H^{-j}, 1 \leq j \leq \ell-1$. More precisely, for $f$ in $H^{-j}$ and $g$ in $H^{j}$, we define (cf. (B.1))

$$
\left\langle R_{\varphi(s)} f, g\right\rangle=\left\langle f, R_{\varphi(s)}^{-1}\left([\operatorname{det}(d \varphi(s))]^{-1} g\right)\right\rangle
$$

where $\langle\cdot, \cdot\rangle$ denotes the extension of the $L^{2}$-inner product $\langle\cdot, \cdot\rangle_{L^{2}}$ as a dual pairing between $H^{-j}$ and $H^{j}$. Indeed, as $\operatorname{det}\left(d \varphi(s) \circ \varphi^{-1}(s)\right)$ is in $H^{\ell-1}$ where $\ell \geq 7$ and $g$ belongs to $H^{j}$, it follows that $R_{\varphi(s)}^{-1}\left([\operatorname{det}(d \varphi(s))]^{-1} g\right)$ is in $H^{j}$ and hence $(6.14)$ is well-defined. Moreover, it follows from (6.14) that for any $|j| \leq \ell-1$ there exist $C_{1}, C_{2}>0$ such that

$$
C_{1}\|f\|_{H^{j}} \leq\left\|R_{\varphi}(s) f\right\|_{H^{j}} \leq C_{2}\|f\|_{H^{j}}
$$


uniformly for $0 \leq s \leq 1$. Finally, the boundedness of $\mathcal{Q}_{k}: H^{-(k-2)} \rightarrow H^{k-2}$ follows from the uniform boundedness of the operators appearing in (6.13). By similar arguments one sees that, more generally, for any $m$ satisfying $2-k \leq m \leq \ell-2 k+1$,

$$
\left.\mathcal{Q}_{k}\right|_{H^{m}}: H^{m} \rightarrow H^{m+2 k-4}
$$

is a bounded linear operator.

Now we establish that $\mathcal{Q}_{k}: H^{-(k-2)} \rightarrow H^{k-2}$ is a linear isomorphism using the Lax-Milgram lemma - see e.g. [23, Chapter 6, Theorem 6]. Consider the bilinear form

$$
\Lambda_{k}: H^{2-k} \times H^{2-k} \rightarrow \mathbb{R}, \quad(f, g) \mapsto\left\langle\mathcal{Q}_{k} f, g\right\rangle
$$

Note that by Lemma B.1 (ii)

$$
\left\langle\mathcal{Q}_{k} f, g\right\rangle=\int_{0}^{1}\left\langle\mathcal{P}_{\lambda}(s) \circ A_{k}^{-1 / 2} \circ R_{\varphi(s)}^{-1} \circ \Psi^{s} f, \mathcal{P}_{\lambda}(s) \circ A_{k}^{-1 / 2} \circ R_{\varphi(s)}^{-1} \circ \Psi^{s} g\right\rangle_{L^{2}} d s .
$$

As $\mathcal{Q}_{k}: H^{-(k-2)} \rightarrow H^{k-2}$ is bounded, there exists a constant $C>0$ such that for any $f, g \in H^{2-k}$,

$$
\left|\left\langle\mathcal{Q}_{k} f, g\right\rangle\right| \leq C\|f\|_{H^{2-k}}\|g\|_{H^{2-k}} .
$$

To see that $\Lambda_{k}$ is positive definite, note that by Lemma B.2, there exists a constant $C>0$ such that for any $0 \leq s \leq 1$ and $f$ in $H^{2},\left\|\mathcal{P}_{\lambda}(s) f\right\|_{L^{2}} \geq C\|f\|_{H^{2}}$. Hence, for any $f$ in $H^{2-k}$,

$$
\begin{aligned}
\left\langle\mathcal{Q}_{k} f, f\right\rangle_{L^{2}} & =\int_{0}^{1}\left\|\mathcal{P}_{\lambda}(s) \circ A_{k}^{-1 / 2} \circ R_{\varphi(s)}^{-1} \circ \Psi^{s} f\right\|_{L^{2}}^{2} d s \\
& \geq C \int_{0}^{1}\left\|A_{k}^{-1 / 2} \circ R_{\varphi(s)}^{-1} \circ \Psi^{s} f\right\|_{H^{2}}^{2} d s \\
& \geq C_{1} \int_{0}^{1}\left\|R_{\varphi(s)}^{-1} \circ \Psi^{s} f\right\|_{H^{2-k}}^{2} d s \\
& \geq C_{2}\|f\|_{H^{2-k}}^{2}
\end{aligned}
$$

for suitably chosen positive constants $C_{1}, C_{2}>0$. Hence, by the Lax-Milgram lemma, we conclude that $\mathcal{Q}_{k}: H^{2-k} \rightarrow H^{k-2}$ is a linear isomorphism.

Next, we use a bootstrapping argument to check that $\mathcal{Q}_{k}: H^{m} \rightarrow H^{m+2 k-4}$ is a linear isomorphism for any $2-k \leq m \leq \ell-2 k+1$. We already know that $\mathcal{Q}_{k}: H^{m} \rightarrow H^{m+2 k-4}$ is a bounded linear operator and, by the previous step, that it is one-to-one. To show that it is onto, we argue by induction with respect to $m$. The case $m=2-k$ has been treated above. Suppose that for an arbitrary $m$ verifying $2-k \leq m \leq \ell-2 k$, we have that $\mathcal{Q}_{k}: H^{m} \rightarrow H^{m+2 k-4}$ is onto (and hence a bijection). We have to show that $\mathcal{Q}_{k}: H^{m+1} \rightarrow H^{m+2 k-3}$ is onto. By the induction hypothesis, for any given $f$ in $H^{m+2 k-3}$, there exists (a unique) $q$ in $H^{m}$ such that $\mathcal{Q}_{k} q=f$. Then, $q=\mathcal{Q}_{k}^{-1} f$, and for $j=1,2$, one has

$$
\partial_{x_{j}} q=\partial_{x_{j}} \mathcal{Q}_{k}^{-1} f=\mathcal{Q}_{k}^{-1} \partial_{x_{j}} f+\left[\partial_{x_{j}}, \mathcal{Q}_{k}^{-1}\right] f .
$$

As $\left[\partial_{x_{j}}, \mathcal{Q}_{k}^{-1}\right]=\mathcal{Q}_{k}^{-1}\left[\mathcal{Q}_{k}, \partial_{x_{j}}\right] \mathcal{Q}_{k}^{-1}$ we get

$$
\partial_{x_{j}} q=\mathcal{Q}_{k}^{-1} \partial_{x_{j}} f+\mathcal{Q}_{k}^{-1}\left[\mathcal{Q}_{k}, \partial_{x_{j}}\right] \mathcal{Q}_{k}^{-1} f .
$$


The first term on the r.h.s. of the latter identity is in $H^{m}$. To see that the second term also lies in $H^{m}$ we write $\mathcal{Q}_{k} h$ for $h \in H^{r}$ with $2-k \leq r \leq \ell-2 k$ as

$$
\mathcal{Q}_{k} h=\int_{0}^{1}\left(\Phi^{s}\right)^{-1} \circ \mathcal{C}_{s}\left(A_{k}^{-1 / 2}\right) \circ \mathcal{C}_{s}\left(\mathcal{P}_{\lambda}^{\dagger}(s)\right) \circ \mathcal{C}_{s}\left(\mathcal{P}_{\lambda}(s)\right) \circ \mathcal{C}_{s}\left(A_{k}^{-1 / 2}\right) \circ \Psi^{s} h d s
$$

where $\mathcal{C}_{s} \equiv \mathcal{C}_{\varphi(s)}$ denotes conjugation of an operator by $R_{\varphi(s)}$,

$$
\mathcal{C}_{s}(\cdot)=R_{\varphi(s)} \circ(\cdot) \circ R_{\varphi(s)}^{-1},
$$

and the integration is performed in $H^{r+2 k-4}$. Using (6.16) and the commutator identity $[a, b c]=[a, b] c+b[a, c]$ valid for any elements of a ring one sees that $\left[\mathcal{Q}_{k}, \partial_{x_{j}}\right] g$ with $g \in H^{m}$ can be represented as a sum of integrals each of which involves the commutator of $\partial_{x_{j}}$ with one of the operators occuring in the integrand of (6.16). One then verifies that each of these summands is an element in $H^{m+(2 k-4)}$ and hence

$$
\mathcal{Q}_{k}^{-1}\left[\mathcal{Q}_{k}, \partial_{x_{j}}\right] \mathcal{Q}_{k}^{-1} f \in H^{m} .
$$

To illustrate how this is done let us consider, for example, the commutator $\left[\mathcal{C}_{s}\left(A_{k}^{-1 / 2}\right), \partial_{x_{j}}\right]$. One has, with $\tilde{\mathcal{C}}_{s}=\mathcal{C}_{\varphi(s)^{-1}}$,

$$
\begin{aligned}
& {\left[\mathcal{C}_{s}\left(A_{k}^{-1 / 2}\right), \partial_{x_{j}}\right]=\left[\mathcal{C}_{s}\left(A_{k}^{-1 / 2}\right), \mathcal{C}_{s}\left(\tilde{\mathcal{C}}_{s}\left(\partial_{x_{j}}\right)\right)\right]=} \\
& =\mathcal{C}_{s}\left(\left[A_{k}^{-1 / 2}, \tilde{\mathcal{C}}_{s}\left(\partial_{x_{j}}\right)\right]\right)=\mathcal{C}_{s}\left(A_{k}^{-1 / 2}\left[\tilde{\mathcal{C}}_{s}\left(\partial_{x_{j}}\right), A_{k}^{1 / 2}\right] A_{k}^{-1 / 2}\right)
\end{aligned}
$$

A direct computation shows that $\tilde{\mathcal{C}}_{s}\left(\partial_{x_{j}}\right)=\sum_{1 \leq l \leq 2} r_{j l}(s) \partial_{x_{l}}$ where $r_{j l}(s)=\partial_{x_{j}} \varphi_{l}(s) \circ$ $\varphi(s)^{-1}$ is in $C^{0}\left((-2,2), H^{\ell-1}\right)$. Hence,

$$
\left[\tilde{\mathcal{C}}_{s}\left(\partial_{x_{j}}\right), A_{k}^{1 / 2}\right]=\sum_{l}\left[A_{k}^{1 / 2}, r_{j l}\right] \partial_{x_{l}}
$$

and by Lemma B.3, for any $k-\ell+2 \leq n \leq \ell-1$, $\left[\tilde{\mathcal{C}}_{s}\left(\partial_{x_{j}}\right), A_{k}^{1 / 2}\right]: H^{n} \rightarrow H^{n-k}$ is a bounded operator whose norm depends continuously on $s \in(-2,2)$. In particular, (6.18) implies that for any $2-\ell \leq n \leq \ell-k-1$

$$
\left[\mathcal{C}_{s}\left(A_{k}^{-1 / 2}\right), \partial_{x_{j}}\right]: H^{n} \rightarrow H^{n+k}
$$

is a bounded operator depending strongly continuously on $s \in(-2,2)$. Hence, for any $g \in H^{m}$ with $2-\ell \leq m \leq \ell-k-1$,

$$
\int_{0}^{1}\left(\Phi^{s}\right)^{-1} \circ \mathcal{C}_{s}\left(A_{k}^{-1 / 2}\right) \circ \mathcal{C}_{s}\left(\mathcal{P}_{\lambda}^{\dagger}(s) \circ \mathcal{P}_{\lambda}(s)\right) \circ\left[\mathcal{C}_{s}\left(A_{k}^{-1 / 2}\right), \partial_{x_{j}}\right] \circ \Psi^{s} g d s
$$

and

$$
\int_{0}^{1}\left(\Phi^{s}\right)^{-1} \circ\left[\mathcal{C}_{s}\left(A_{k}^{-1 / 2}\right), \partial_{x_{j}}\right] \circ \mathcal{C}_{s}\left(\mathcal{P}_{\lambda}^{\dagger}(s) \circ \mathcal{P}_{\lambda}(s)\right) \circ \mathcal{C}_{s}\left(A_{k}^{-1 / 2}\right) \circ \Psi^{s} g d s
$$

belong to $H^{m+2 k-4}$. In a straightforward way one sees that for any $4-\ell \leq m \leq \ell-1$, $\left[\mathcal{C}_{s}\left(\mathcal{P}_{\lambda}(s)\right), \partial_{x_{j}}\right]: H^{m} \rightarrow H^{m-2}$ and $\left[\mathcal{C}_{s}\left(\mathcal{P}_{\lambda}^{\dagger}(s)\right), \partial_{x_{j}}\right]: H^{m} \rightarrow H^{m-2}$, whereas for any $2-\ell \leq m \leq \ell-2,\left[\Psi^{s}, \partial_{x_{j}}\right]: H^{m} \rightarrow H^{m}$ and $\left[\left(\Phi^{s}\right)^{-1}, \partial_{x_{j}}\right]: H^{m} \rightarrow H^{m}$, are bounded operators depending (strongly) continuously on $s \in(-2,2)$. Hence all 
integrals appearing in the above mentioned representation of $\left[\mathcal{Q}_{k}, \partial_{x_{j}}\right] g$ are elements in $H^{m+2 k-4}$ and (6.17) holds as claimed.

Now (6.17) implies that, for $j=1,2$,

$$
\partial_{x_{j}} q=\mathcal{Q}_{k}^{-1} \partial_{x_{j}} f+\mathcal{Q}_{k}^{-1}\left[\mathcal{Q}_{k}, \partial_{x_{j}}\right] \mathcal{Q}_{k}^{-1} f
$$

is in $H^{m}$, and therefore $q$ belongs to $H^{m+1}$. This shows that $\mathcal{Q}_{k}: H^{m+1} \rightarrow H^{m+2 k-3}$ is onto and hence a linear isomorphism. This completes the induction argument.

In particular, $\mathcal{Q}_{k}: H^{\ell+1-2 k} \rightarrow H^{\ell-3}$ is a linear isomorphism. As $\varphi(1)$ is assumed to be in $\mathcal{D}_{+}^{\ell+1}$, it then follows from (6.12) that

$$
A_{k} v_{0}=\mathcal{Q}_{k}^{-1}\left(\Phi^{1}\right)^{-1}\left(\Delta^{2} \varphi(1)+\ldots\right)
$$

is in $H^{\ell+1-2 k}$ i.e., that $v_{0}$ is in $H^{\ell+1}$.

To prove Proposition 5.2 we need to make some preparations. The following arguments are valid for $v_{0}$ in $V_{\ell+2}^{(k)}$ where $\ell \geq \ell_{k}$. So let us assume that $v_{0}$ is in $V_{\ell+2}^{(k)}$. It follows by Theorem 4.1 that the solution $(\varphi, v)$ of (1.2) issuing form (id, $\left.v_{0}\right)$ is in $C^{1}\left((-2,2), \mathcal{D}_{+}^{\ell+2} \times H^{\ell+2}\right)$ and that the corresponding solution $u=v \circ \varphi^{-1}$ of $(1.7)$ is in $C^{0}\left((-2,2), H^{\ell+2}\right) \cap C^{1}\left((-2,2), H^{\ell+1}\right)$. Now, let us compute the derivative $\delta \varphi(t):=$ $d_{v_{0}} \operatorname{Exp}_{k, \ell}\left(\delta v_{0}\right)$ of $\operatorname{Exp}_{k, \ell}$ at the point $v_{0}$ in the direction $\delta v_{0} \in H^{\ell}$. For this purpose introduce $\delta v(t):=\left.\frac{d}{d \varepsilon}\right|_{\varepsilon=0} v\left(t ; v_{0}+\varepsilon \delta v_{0}\right)$ and $\delta u:=\delta u(t)=\left.\frac{d}{d \varepsilon}\right|_{\varepsilon=0} u\left(t ; v_{0}+\varepsilon \delta v_{0}\right)$. By Remark 4.2 one has $(\delta \varphi, \delta v) \in C^{0}\left((-2,2), H^{\ell} \times H^{\ell}\right)$. Moreover, the variation of (1.6) in $H^{\ell-1}$ leads to

$$
\delta u=(\delta v) \circ \varphi^{-1}+\left[(d v) \circ \varphi^{-1}\right] \delta \varphi^{-1},
$$

where $\delta \varphi^{-1} \circ \varphi=-(d \varphi)^{-1} \delta \varphi$, so that

$$
\delta u=\left[\delta v-(d v)(d \varphi)^{-1} \delta \varphi\right] \circ \varphi^{-1} .
$$

Using that $(\varphi, v)$ is in $C^{1}\left((-2,2), \mathcal{D}_{+}^{\ell+2} \times H^{\ell+2}\right)$, one concludes that $\delta u \in$ $C^{0}\left((-2,2), H^{\ell}\right)$. The variation of the integral analogue of (1.6),

$$
\varphi(t)=\mathrm{id}+\int_{0}^{t} u \circ \varphi(s) d s
$$

leads to

$$
(\delta \varphi)(t)=\int_{0}^{1}[\delta u \circ \varphi+(d u \circ \varphi) \delta \varphi] d s
$$

where the integration is performed in $H^{\ell-1}$. As the integrand in the later formula lies in $C^{0}\left((-2,2), H^{\ell}\right)$, we see that $\delta \varphi \in C^{1}\left((-2,2), H^{\ell}\right)$ and satisfies the following inhomogeneous linear equation in $H^{\ell}$,

$$
\left\{\begin{array}{l}
(\delta \varphi)^{\cdot}-[(d u) \circ \varphi] \delta \varphi=\delta u \circ \varphi \\
\delta \varphi(0)=0
\end{array}\right.
$$

It follows from (6.19) and the method of the variation of parameters that

$$
\delta \varphi(t)=\Phi^{t} \int_{0}^{t}\left(\Phi^{s}\right)^{-1}(\delta u(s) \circ \varphi(s)) d s
$$


where, $\Phi^{t}=\Phi^{t}(x)$ is the $2 \times 2$ fundamental matrix solution of (6.1) and the integration is performed in $H^{\ell}$. To express the r.h.s. of (6.20) in terms of the initial data, we need to investigate the inhomogeneous term on the r.h.s of (6.19). To this end, passing to the integral version of (1.7) and arguing as above we get, after composing with $\varphi$,

$$
\left\{\begin{array}{l}
{\left[\left(A_{k} \delta u\right) \circ \varphi\right]^{\cdot}+\left\{\left[\operatorname{div} u \cdot \operatorname{Id}+(d u)^{\dagger}\right] \circ \varphi\right\}\left[\left(A_{k} \delta u\right) \circ \varphi\right]=g_{k}} \\
A_{k} \delta u(0)=A_{k} \delta v_{0}
\end{array}\right.
$$

where

$$
g_{k}:=-\left\{\left(d\left(A_{k} u\right)\right) \delta u+\left[\operatorname{div}(\delta u) \cdot \operatorname{Id}+(d(\delta u))^{\dagger}\right] A_{k} u\right\} \circ \varphi .
$$

Note that $g_{k}$ contains only derivatives of $\delta u$ up to first order. Hence, by the regularity properties of $\varphi, u$, and $\delta u$ discussed above, and the crucial assumption that $k \geq 1$, $g_{k}$ is in $C^{0}\left((-2,2), H^{\ell+1-2 k}\right)$. Equation (6.21a) is a linear inhomogeneous ODE for $\left(A_{k} \delta u\right) \circ \varphi$. Hence, the solution of $(6.21 \mathrm{a})$ is given by

$$
\left(A_{k} \delta u(s)\right) \circ \varphi(s)=\Psi^{s}\left(A_{k} \delta v_{0}+\int_{0}^{s}\left(\Psi^{\tau}\right)^{-1} g_{k}(\tau) d \tau\right)
$$

where $\Psi^{s}=\Psi^{s}(x)$ is the $2 \times 2$ fundamental matrix solution of (6.3) and the integration is carried out in $H^{\ell+1-2 k}$. Solving (6.22) for $\delta u(s)$ we get

$$
\delta u(s) \circ \varphi(s)=R_{\varphi(s)} \circ A_{k}^{-1} \circ R_{\varphi(s)}^{-1}\left(\Psi^{s}\left(A_{k} \delta v_{0}+\int_{0}^{s}\left(\Psi^{\tau}\right)^{-1} g_{k}(\tau) d \tau\right)\right) .
$$

We need to investigate the regularity of the r.h.s. of (6.23). As $v_{0}$ is assumed to be in $V_{\ell+2}^{(k)}$, the coefficients of the matrices $d u \circ \varphi$ and [div $\left.u \cdot \operatorname{Id}+(d u)^{\dagger}\right] \circ \varphi$ of $(6.1)$ and $(6.3)$ are in $C^{0}\left((-2,2), H^{\ell+1}\right)$. In particular, $d u \circ \varphi$ and [div $\left.u \cdot \operatorname{Id}+(d u)^{\dagger}\right] \circ \varphi$ are in $C^{0}\left((-2,2), \mathcal{L}\left(H^{\ell+1}, H^{\ell+1}\right)\right)$ and hence, by the ODE theory, the columns of the $2 \times 2$ matrices $\Phi$ and $\Psi$ are in $C^{1}\left((-2,2), H^{\ell+1}\right)$. It then follows from (6.23) that

$$
\delta u(s) \circ \varphi(s)=R_{\varphi(s)} \circ A_{k}^{-1} \circ R_{\varphi(s)}^{-1} \circ \Psi^{s}\left(A_{k} \delta v_{0}\right)+\ldots
$$

where $\ldots$ stand for terms in $C^{0}\left((-2,2), H^{\ell+1}\right)$. Hence, by $(6.20)$,

$$
\begin{aligned}
\delta \varphi(1) & =\Phi^{1} \int_{0}^{1}\left(\Phi^{s}\right)^{-1}(\delta u(s) \circ \varphi(s)) d s \\
& =\Phi^{1} \int_{0}^{1}\left(\Phi^{s}\right)^{-1} \circ R_{\varphi(s)} \circ A_{k}^{-1} \circ R_{\varphi(s)}^{-1} \circ \Psi^{s}\left(A_{k} \delta v_{0}\right) d s+\ldots \\
& =\Phi^{1} \mathcal{R}_{k}\left(A_{k} \delta v_{0}\right)+\ldots
\end{aligned}
$$

where $\ldots$ stand for terms in $C^{0}\left((-2,2), H^{\ell+1}\right)$ and for $f \in H^{\ell-2 k}$

$$
\mathcal{R}_{k} f:=\int_{0}^{1}\left(\Phi^{s}\right)^{-1} \circ R_{\varphi(s)} \circ A_{k}^{-1 / 2} \circ A_{k}^{-1 / 2} \circ R_{\varphi(s)}^{-1} \circ \Psi^{s} f d s .
$$

Notice that the operator $\mathcal{R}_{k}$ is of the same form as the operator $\mathcal{Q}_{k}$ defined in (6.13). In particular, arguing as in the proof of Proposition 5.1, one sees that $\mathcal{R}_{k}$ extends to an operator $\mathcal{R}_{k}: H^{-k} \rightarrow H^{k}$ and for any $-k \leq m \leq \ell-2 k+1$

$$
\left.\mathcal{R}_{k}\right|_{H^{m}}: H^{m} \rightarrow H^{m+2 k}
$$


is a linear isomorphism.

Proof of Proposition 5.2. Assume that $v_{0} \in V_{\ell+2}^{(k)}$. Then since (6.27) is a linear isomorphism for $m=\ell-2 k$ and $\ell-2 k+1$ and since the matrix $\Phi^{1}$ is invertible with elements in $H^{\ell+1}$ we get from (6.25) that $\delta v_{0}$ is in $H^{\ell} \backslash H^{\ell+1}$ if and only if $\delta \varphi(1)$ is in $H^{\ell} \backslash H^{\ell+1}$. In other words, we have shown that

$$
d_{v_{0}} \operatorname{Exp}_{k, \ell}\left(\delta v_{0}\right) \in H^{\ell} \backslash H^{\ell+1} \quad \text { if and only if } \quad \delta v_{0} \in H^{\ell} \backslash H^{\ell+1}
$$

$\square$

7. The exponential map for $k=0$. In this section we prove Theorem 1.5. It says that even if we assume that the exponential map $\operatorname{Exp}_{0}$ can be defined near zero it does not define a $C_{F}^{1}$-chart of id in $\mathcal{D}_{+}$.

Proof of Theorem 1.5. We follow the idea of the proof of the corresponding result for the circle in [7], [8]. For $k=0,(1.7)$ takes the form

$$
\left\{\begin{array}{l}
\dot{u}+\left(d u+(d u)^{\dagger}+\operatorname{div} u \cdot \operatorname{Id}\right) u=0 \\
u(0)=v_{0}
\end{array}\right.
$$

Consider the initial data $v_{0}(x)=c e_{1}$ in which $e_{1}=(1,0)$, and $c$ in $\mathbb{R} \backslash\{0\}$. Then $u(t, x) \equiv c e_{1}$ is a global (in time) solution of (7.1). The equation for the geodesic flow (1.6) then reads

$$
\dot{\varphi}=c e_{1}, \varphi(0)=\mathrm{id}
$$

so that

$$
\varphi(t, x)=x+c t e_{1}
$$

By assumption, the exponential map $\operatorname{Exp}_{0}$ is uniquely defined near 0; hence, it follows that for $c$ sufficiently small

$$
\operatorname{Exp}_{0}\left(c e_{1}\right)(x)=\varphi(1, x)=x+c e_{1}, \forall x \in \mathbb{T}^{2} .
$$

In view of Remark A.4, Theorem 1.5 will follow if we can show that there exists $c$ in $\mathbb{R} \backslash\{0\}$ arbitrarily close to 0 so that

$$
d_{c e_{1}} \operatorname{Exp}_{0}: C^{\infty}\left(\mathbb{T}^{2}, \mathbb{R}^{2}\right) \rightarrow T_{\mathrm{id}+c e_{1}} \mathcal{D}_{+}
$$

is not one-to-one. To this end, we consider the variational equations for $\delta u$ and $\delta \varphi$, obtained by linearizing equations (7.1) and (7.2) at the solutions $u(t, x)=c e_{1}$, and $\varphi(t, x)=x+$ cte $_{1}$ respectively. One gets

$$
\left\{\begin{array}{l}
(\delta u)^{\cdot}+c\left[d(\delta u)+(d(\delta u))^{\dagger}+\operatorname{div}(\delta u) \cdot \operatorname{Id}\right] e_{1}=0 \\
\delta u(0)=\delta v_{0}
\end{array}\right.
$$

and

$$
\left\{\begin{array}{l}
(\delta \varphi)^{\cdot}=\delta u\left(t, x+c t e_{1}\right) \\
\delta \varphi(0)=0
\end{array}\right.
$$


In particular, for a variation of the form $\delta v_{0}=h e_{1}$, with $h(x):=h\left(x_{1}\right)$, we have that componentwise (7.3a) is given by

$$
\left\{\begin{array}{lll}
\left(\delta u_{1}\right)^{\cdot}+c\left(3 \partial_{x_{1}}\left(\delta u_{1}\right)+\partial_{x_{2}}\left(\delta u_{2}\right)\right) & =0, & \delta u_{1}(0)=h \\
\left(\delta u_{2}\right)^{\cdot}+c\left(\partial_{x_{1}}\left(\delta u_{2}\right)+\partial_{x_{2}}\left(\delta u_{1}\right)\right) & =0, & \delta u_{2}(0)=0 .
\end{array}\right.
$$

A solution is $\delta u(t, x)=h\left(x_{1}-3 c t\right) e_{1}$. Hence the corresponding solution of $(7.3 \mathrm{~b})$ is

$$
\delta \varphi(t, x)=\int_{0}^{t} h\left(x_{1}-2 c s\right) d s \cdot e_{1}
$$

and thus

$$
\left(d_{c e_{1}} \operatorname{Exp}_{0}\right)\left(h e_{1}\right)(x)=\int_{0}^{1} h\left(x_{1}-2 c s\right) d s \cdot e_{1}
$$

Now consider $c_{\ell}:=\frac{1}{\ell}$, and let $h_{\ell}\left(x_{1}\right):=\cos \left(2 \pi \ell x_{1}\right)$, with $\ell$ in $\mathbb{Z}_{\geq 1}$. Then

$$
\int_{0}^{1} h_{\ell}\left(x_{1}-2 c_{\ell} s\right) d s=\int_{0}^{1} \cos \left(2 \pi\left(\ell x_{1}-2 s\right)\right) d s=0,
$$

i.e.,

$$
\left(d_{\frac{1}{\ell} e_{1}} \operatorname{Exp}_{0}\right)\left(h_{\ell} e_{1}\right)=0, \quad \forall \ell \in \mathbb{Z}_{\geq 1} .
$$

Hence, by Remark A.4, the map $\operatorname{Exp}_{0}$ cannot be a local $C_{F}^{1}$-diffeomorphism near the origin in $T_{\mathrm{id}} \mathcal{D}_{+} \cdot \square$

REMARK 7.1. Note that the group $\mathcal{D}_{+}(\mathbb{T})$ of smooth orientation preserving diffeomorphisms of the circle $\mathbb{T}$ can be canonically embedded into $\mathcal{D}_{+}\left(\mathbb{T}^{2}\right)$,

$$
\mathcal{D}_{+}(\mathbb{T}) \rightarrow \mathcal{D}_{+}\left(\mathbb{T}^{2}\right), \varphi_{1} \mapsto \varphi_{1} \times \mathrm{id} .
$$

It is straightforward to verify that $\mathcal{D}_{+}(\mathbb{T})$ is a totally geodesic Fréchet submanifold of $\mathcal{D}_{+}\left(\mathbb{T}^{2}\right)$ with respect to the right invariant metric $\nu^{(0)}$. In this way, Theorem 1.5 then follows from the corresponding result for $\mathcal{D}_{+}(\mathbb{T})$ (cf. [7], [8].)

Appendix A. Calculus on Fréchet spaces. For the convenience of the reader we recall in this appendix some definitions and notions from the calculus in Fréchet spaces put together in [6], and present an inverse function theorem valid in a set-up for Fréchet spaces which is suitable for our purposes. For more details we refer the reader to [14] and [21].

Fréchet spaces: Consider the pair $\left(X,\left\{\|\cdot\|_{n}\right\}_{n \geq 0}\right)$ where $X$ is a real vector space and $\left\{\|\cdot\|_{n}\right\}_{n \geq 0}$ is a countable collection of seminorms. A topology on $X$ is defined in the usual way as follows: A basis of open neighborhoods of $0 \in X$ is given by the sets

$$
U_{\epsilon, k_{1}, \ldots, k_{s}}:=\left\{x \in X:\|x\|_{k_{j}}<\epsilon \forall 1 \leq j \leq s\right\}
$$

where $s, k_{1}, \ldots k_{s} \in \mathbb{Z}_{\geq 0}$ and $\epsilon>0$. Then the topology on $X$ is defined as the collection of open sets generated by the sets $x+U_{\epsilon, k_{1}, \ldots, k_{s}}$, for arbitrary $x$ in $X$ and arbitrary 
$s, k_{1}, \ldots, k_{s} \in \mathbb{Z}_{>0}$ and $\epsilon>0$. In this way $X$ becomes a topological vector space. Note that a sequence $x_{k}$ converges to $x$ in $X$ iff for any $n \geq 0,\left\|x_{k}-x\right\|_{n} \rightarrow 0$ as $k \rightarrow+\infty$.

Moreover, the topological vector space $X$ described above is Hausdorff iff for any $x$ in $X,\|x\|_{n}=0$ for every $n$ in $\mathbb{Z}_{\geq 0}$ implies $x=0$. A sequence $\left(x_{k}\right)_{k \in \mathbb{N}}$ is called Cauchy iff it is a Cauchy sequence with respect to any of the seminorms. By definition, $X$ is complete iff every Cauchy sequence converges in $X$.

Definition A.1. A pair $\left(X,\left\{\|\cdot\|_{n}\right\}_{n \geq 0}\right)$ consisting of a topological vector space $X$ and a countable system of seminorms $\left\{\|\cdot\|_{n}\right\}_{n \geq 0}$ is called a Fréchet space ${ }^{2}$ iff the topology of $X$ is the one induced by $\left\{\|\cdot\|_{n}\right\}_{n \geq 0}$, and $X$ is Hausdorff and complete.

$C_{F}^{1}$-differentiability: Let $f: U \subseteq X \rightarrow Y$ be a map from an open set $U$ of a Fréchet space $X$ to a Fréchet space $Y$.

Definition A.2. If the limit

$$
\lim _{\epsilon \rightarrow 0} \frac{1}{\epsilon}(f(x+\epsilon h)-f(x))
$$

in $Y$ exists with respect to the Fréchet topology of $Y$, we say that $f$ is differentiable at $x$ in the direction $h$. The limit is declared to be the directional derivative of $f$ at the point $x$ in $U$ in the direction $h$ in $X$, and is denoted by $\delta_{x} f(h)$.

Definition A.3. If the directional derivative $\delta_{x} f(h)$ exists for any $x$ in $U$ and any $h$ in $X$, and the map

$$
(x, h) \mapsto \delta_{x} f(h), U \times X \rightarrow Y
$$

is continuous with respect to the Fréchet topology on $U \times X$ and $Y$, then $f$ is called continuously differentiable on $U$ or $C_{F}^{1}$-smooth. The space of all such maps is denoted by $C_{F}^{1}(U, Y){ }^{3} A$ map $f: U \rightarrow V$ from an open set $U \subseteq X$ onto an open set $V \subseteq Y$ is called a $C_{F}^{1}$-diffeomorphism if $f$ is a homeomorphism and $f$ as well as $f^{-1}$ are $C_{F}^{1}$-smooth.

REMARK A.4. Using the chain rule one easily obtains that for any $x$ in $U$ the directional derivative $\delta_{x} f: X \rightarrow Y$ of a $C_{F}^{1}$-diffeomorphism $f: U \rightarrow V$ is a linear isomorphism.

In this paper we consider mainly the following spaces:

Fréchet space $C^{\infty}\left(\mathbb{T}^{2}, \mathbb{R}^{2}\right)$. The space $C^{\infty}\left(\mathbb{T}^{2}, \mathbb{R}^{2}\right)$ denotes the real vector space of $C^{\infty}$-smooth, functions $u: \mathbb{T}^{2} \rightarrow \mathbb{R}^{2}$. The topology on $C^{\infty}\left(\mathbb{T}^{2}, \mathbb{R}^{2}\right)$ is induced by the countable system of Sobolev norms:

$$
\|u\|_{n}:=\|u\|_{H^{n}}=\left(\sum_{j=0}^{n} \int_{\mathbb{T}^{2}}\left\langle(-\Delta)^{j} u, u\right\rangle d x\right)^{1 / 2}, \quad n \geq 0 .
$$

\footnotetext{
${ }^{2}$ Unlike for the standard notion of a Fréchet space, here the countable system of seminorms defining the topology of $X$ is part of the structure of the space.

${ }^{3}$ Note that even in the case where $X$ and $Y$ are Banach spaces this definition of continuous differentiability is weaker than the usual one (cf. [14]). In order to distinguish it from the classical one we write $C_{F}^{1}$ instead of $C^{1}$. We refer to [14] for a discussion of the reasons to introduce the notion of $C_{F}^{1}$-differentiability.
} 
Fréchet manifold $\mathcal{D}_{+}\left(\mathbb{T}^{2}\right)$. By definition, $\mathcal{D}_{+}=\mathcal{D}_{+}\left(\mathbb{T}^{2}\right)$ denotes the group of $C^{\infty}$-smooth positively oriented diffeomorphisms of the 2 -d torus $\mathbb{T}^{2}=(\mathbb{R} / \mathbb{Z})^{2}$. A Fréchet manifold structure on $\mathcal{D}_{+}$can be introduced as follows: Passing in domain and target to the universal cover $\mathbb{R}^{2} \rightarrow \mathbb{T}^{2}$ of $\mathbb{T}^{2}$, any element $\varphi$ of $\mathcal{D}_{+}$gives rise to a smooth diffeomorphism of $\mathbb{R}^{2}$ in $C^{\infty}\left(\mathbb{R}^{2}, \mathbb{R}^{2}\right)$, again denoted by $\varphi$, where each of the components $\varphi_{j}, j=1,2$, satisfies the normalization conditions

$$
\mathrm{C}_{1}\left(\varphi_{j}\right):-1 / 2<\varphi_{j}(0)<1 / 2 \quad \text { or } \quad \mathrm{C}_{2}\left(\varphi_{j}\right): 0<\varphi_{j}(0)<1 .
$$

Note that for any $k \in \mathbb{Z}^{2}, \varphi(x+k)-\varphi(x)$ is a continuous function of $x$ with values in $\mathbb{Z}^{2}$. Hence, it is independent of $x \in \mathbb{R}^{2}$. Then, by a similar argument, the linear map

$$
T_{\varphi}: \mathbb{R}^{2} \rightarrow \mathbb{R}^{2}, \quad\left(x_{1}, x_{2}\right) \mapsto x_{1}(\varphi(1,0)-\varphi(0,0))+x_{2}(\varphi(0,1)-\varphi(0,0))
$$

has the property that the function $f:=\varphi-T_{\varphi}$ is 1-periodic in $x_{1}$ and $x_{2}$, and hence lies in $C^{\infty}\left(\mathbb{T}^{2}, \mathbb{R}^{2}\right)$. Note that $T_{\varphi} \in S L(2 ; \mathbb{Z})$ and $\operatorname{det}\left(T_{\varphi}+d_{x} f\right)>0$ at any $x$ in $\mathbb{R}^{2}$. The normalizations (A.1) give rise to the following atlas of charts $\left\{\mathfrak{U}_{i, j, T}\right\}_{1 \leq i, j \leq 2, T \in S L(2 ; \mathbb{Z})}$ of $\mathcal{D}_{+}$, with $\bigcup_{i, j, T} \mathfrak{U}_{i, j, T}=\mathcal{D}_{+}$, defined by

$$
\mathfrak{F}_{i, j, T}: \mathfrak{V}_{i, j, T} \rightarrow \mathfrak{U}_{i, j, T}, \quad f \mapsto \varphi:=T+f
$$

where $\mathfrak{U}_{i, j, T}=T+\mathfrak{V}_{i, j, T}$, and

$$
\mathfrak{V}_{i, j, T}:=\left\{f \in C^{\infty}\left(\mathbb{T}^{2}, \mathbb{R}^{2}\right): C_{i}\left(f_{1}\right) ; C_{j}\left(f_{2}\right) ; \operatorname{det}(T+d f)>0\right\} .
$$

As $\mathfrak{V}_{i, j, T}(1 \leq i, j \leq 2, T \in S L(2 ; \mathbb{Z}))$ are open subsets in the Fréchet space $C^{\infty}\left(\mathbb{T}^{2}, \mathbb{R}^{2}\right)$, the construction above gives an atlas of Fréchet charts of $\mathcal{D}_{+}$. In this way, $\mathcal{D}_{+}$is a Fréchet manifold modeled on $C^{\infty}\left(\mathbb{T}^{2}, \mathbb{R}^{2}\right)$.

Hilbert manifold $\mathcal{D}_{+}^{\ell}\left(\mathbb{T}^{2}\right)(\ell \geq 3)$. $\mathcal{D}_{+}^{\ell}=\mathcal{D}_{+}^{\ell}\left(\mathbb{T}^{2}\right)$ denotes the group of positively oriented $C^{1}$-diffeomorphisms of $\mathbb{T}^{2}$ of class $H^{\ell}$. By definition, a $C^{1}$-diffeomorphism $\varphi$ of $\mathbb{T}^{2}$ is in $H^{\ell}$ iff any lift of $\varphi$ is in $H_{\text {loc }}^{\ell}\left(\mathbb{R}^{2}, \mathbb{R}^{2}\right)$. As for $\mathcal{D}_{+}$, one can introduce an atlas for $\mathcal{D}_{+}^{\ell}$ making $\mathcal{D}_{+}^{\ell}$ into a Hilbert manifold modeled on $H^{\ell}$.

Hilbert approximations: Assume that for a given Fréchet space $X$ there is a sequence of Hilbert spaces $\left\{\left(X_{n},\|\cdot\|_{n}\right)\right\}_{n \geq 0}$ such that

$$
X_{0} \supseteq X_{1} \supseteq X_{2} \supseteq \ldots \supseteq X \text { and } X=\bigcap_{n=0}^{\infty} X_{n}
$$

where $\left\{\|\cdot\|_{n}\right\}_{n \geq 0}$ is a sequence of norms inducing the topology on $X$ so that $\|x\|_{0} \leq$ $\|x\|_{1} \leq\|x\|_{2} \leq \ldots$ for any $x$ in $X$. Such a sequence of Hilbert spaces $\left\{\left(X_{n},\|\cdot\|_{n}\right)\right\}_{n \geq 0}$ is called a Hilbert approximation of the Fréchet space $X$. For Fréchet spaces admitting Hilbert approximations one can prove the following version of the inverse function theorem.

TheOREm A.5. Let $X$ and $Y$ be Fréchet spaces over $\mathbb{R}$ with Hilbert approximations $\left(X_{n},\|\cdot\|_{n}\right)_{n \geq 0}$, and, respectively, $\left(Y_{n},|\cdot|{ }_{n}\right)_{n \geq 0}$. Let $f: V_{0} \rightarrow U_{0}$ be a map between the open subsets $V_{0} \subseteq X_{0}$ and $U_{0} \subseteq Y_{0}$ of the Hilbert spaces $X_{0}$, respectively $Y_{0}$. Define, for any $n \geq 0$,

$$
V_{n}:=V_{0} \cap X_{n}, \quad U_{n}:=U_{0} \cap Y_{n} .
$$

Assume that, for any $n \geq 0$, the following properties are satisfied: 
(a) $f: V_{0} \rightarrow U_{0}$ is a bijective $C^{1}$-map, and, for any $x$ in $V:=V_{0} \cap X, d_{x} f$ : $X_{0} \rightarrow Y_{0}$ is a linear isomorphism;

(b) $f\left(V_{n}\right) \subseteq Y_{n}$, and the restriction $\left.f\right|_{V_{n}}: V_{n} \rightarrow Y_{n}$ is a $C^{1}$-map;

(c) $f\left(V_{n}\right) \supseteq U_{n}$;

(d) for any $x$ in $V, d_{x} f\left(X_{n} \backslash X_{n+1}\right) \subseteq Y_{n} \backslash Y_{n+1}$.

Then for the open subsets $V:=V_{0} \cap X \subseteq X$ and $U:=U_{0} \cap Y \subseteq Y$, one has $f(V) \subseteq U$ and the map $f_{\infty}:=\left.f\right|_{V}: V \rightarrow U$ is a $C_{F}^{1}$-diffeomorphism.

Proof. By properties (a) and (b), $f_{n}:=\left.f\right|_{V_{n}}: V_{n} \rightarrow U_{n}$ is a well-defined, injective $C^{1}$-map. By (c), $f_{n}$ is onto. Hence, $f_{\infty}:=\left.f\right|_{V}: V \rightarrow U$ is bijective. In order to prove that $f_{\infty}: V \rightarrow U$ is a $C_{F}^{1}$-diffeomorphism, consider, for any $n \geq 0$, and any $x$ in $V$, the differential $d_{x} f_{n}: X_{n} \rightarrow Y_{n}$. As $\left.\left(d_{x} f\right)\right|_{X_{n}}=d_{x} f_{n}$ and, by (a), $d_{x} f: X_{0} \rightarrow Y_{0}$ is bijective, one concludes that $d_{x} f_{n}$ is one-to-one. We prove by induction (with respect to $n$ ) that, for any $x$ in $V, d_{x} f_{n}: X_{n} \rightarrow Y_{n}$ is onto. For $n=0\left(V \subseteq V_{0}\right)$, the statement is true by property (a). Next, assume that for arbitrary positive integer $n$, and arbitrary $x$ in $V, d_{x} f_{n-1}: X_{n-1} \rightarrow Y_{n-1}$ is onto. Then, for any $x$ in $V$, and $\eta$ in $Y_{n} \subseteq Y_{n-1}$, there exists a (unique) $\xi$ in $X_{n-1}$ verifying $d_{x} f_{n-1}(\xi)=\eta$. By property (d), it follows that $\xi$ belongs to $X_{n}$. In other words, for any given $n \geq 0$, and any $x$ in $V$, we have that the map $d_{x} f_{n}: X_{n} \rightarrow Y_{n}$ is bijective, and thus, by open mappings theorem, the inverse $\left(d_{x} f_{n}\right)^{-1}: Y_{n} \rightarrow X_{n}$ is a bounded linear operator. As, for any $n \geq 0, f_{n}$ is $C^{1}$-smooth, the map

$$
V_{n}^{\prime} \times X_{n} \rightarrow Y_{n},(x, \xi) \mapsto d_{x} f_{n}(\xi)
$$

is continuous and, by the inverse function theorem it follows that

$$
U_{n}^{\prime} \times Y_{n} \rightarrow X_{n},(y, \eta) \mapsto d_{y}\left(f_{n}^{-1}\right)(\eta)
$$

is continuous as well. Here $V_{n}^{\prime}$ (and $U_{n}^{\prime}$ ) denotes the subset $V$ (respectively $U$ ) with the topology induced by $|\cdot|_{n}$ (respectively $\|\cdot\|_{n}$ ). As for any $x$ in $V$, and $n \geq 0$,

$$
\delta_{x} f_{\infty}=\left.d_{x} f_{n}\right|_{X}
$$

one gets from (A.3) - (A.4) that

$$
V \times X \rightarrow Y,(x, \xi) \rightarrow \delta_{x} f_{\infty}(\xi)
$$

and

$$
U \times Y \rightarrow X,(x, \eta) \mapsto \delta_{y} f_{\infty}^{-1}(\eta)
$$

are continuous. In particular, one concludes (cf. Definition A.3) that

$$
f_{\infty}: V \rightarrow U
$$

is a $C_{F}^{1}$-diffeomorphism.

Appendix B. Auxiliary results. In this appendix we collect some elementary auxiliary results used in the proofs of Propositions 5.1 and 5.2. We freely use the notations introduced in the main body of the paper.

Lemma B.1. Let $(\varphi, v)$ and $u=v \circ \varphi^{-1}$ be the solutions of (1.2), respectively, (1.7) given by Theorem 4.1. Then for any given $x \in \mathbb{T}^{2}$ and $-2<t<2$, 
(i) $\left(\Psi^{t}(x)\right)^{\dagger}=\left[\left(\operatorname{det} \Phi^{t}(x)\right) \Phi^{t}(x)\right]^{-1}$;

(ii) $\left(\left(\Phi^{t}(x)\right)^{-1} \circ R_{\varphi(t, x)}\right)^{\dagger}=R_{\varphi(t, x)}^{-1} \circ \Psi^{t}(x)$,

where $E^{\dagger}$ denotes the conjugation of a linear operator $E: H^{m} \rightarrow H^{m}$ with respect to the $L^{2}$-scalar product extended by continuity to a bilinear pairing $H^{-m} \times H^{m} \rightarrow \mathbb{R}$ $(0 \leq m \leq \ell-1)$.

Proof. (i) From (6.1) and (6.3) it follows that, suppressing $t$ in $\Psi^{t}$ and $\Phi^{t}$,

$$
\Psi^{\dagger} \dot{\Phi}=\Psi^{\dagger}[(d u) \circ \varphi] \Phi=-\left((\dot{\Psi})^{\dagger}+[(\operatorname{div} u) \circ \varphi] \Psi^{\dagger}\right) \Phi,
$$

so that

$$
\left\{\begin{array}{l}
\left(\Psi^{\dagger} \Phi\right)^{\cdot}=-[(\operatorname{div} u) \circ \varphi] \Psi^{\dagger} \Phi \\
\left(\Psi^{\dagger} \Phi\right)^{0}=\mathrm{Id} .
\end{array}\right.
$$

Solving the latter we get, for any given $x \in \mathbb{T}^{2}$, and $-2<t<2$,

$$
\left(\Psi^{t}(x)\right)^{\dagger} \Phi^{t}(x)=e^{-\int_{0}^{t}(\operatorname{div} u)(\tau, \varphi(\tau, x)) d \tau} \mathrm{Id} .
$$

To prove item (i) it remains to show that the exponential factor in the latter identity is given by $\left(\operatorname{det} \Phi^{t}(x)\right)^{-1}$. For any given $x \in \mathbb{T}^{2}$, this relation follows from the Liouville formula for the Wronskian of the linear equation (6.1),

$$
\operatorname{det} \Phi^{t}(x)=e^{\int_{0}^{t} \operatorname{Tr}(d u(\tau, \varphi(\tau, x))) d \tau}=e^{\int_{0}^{t}(\operatorname{div} u)(\tau, \varphi(\tau, x)) d \tau} .
$$

(ii) For any pair $f, g$ of $\mathbb{R}^{2}$-valued $L^{2}$-functions on $\mathbb{T}^{2}$ we have

$$
\begin{aligned}
\left\langle f, R_{\varphi(t)} g\right\rangle_{L^{2}} & =\left\langle R_{\varphi(t)}^{-1} f,\left[\operatorname{det}\left(d \varphi^{-1}(t)\right)\right] g\right\rangle_{L^{2}} \\
& =\left\langle R_{\varphi(t)}^{-1}\left([\operatorname{det}(d \varphi(t))]^{-1} f\right), g\right\rangle_{L^{2}} .
\end{aligned}
$$

The first identity in the above display follows from an obvious change of variables. The second one follows from the fact that $\left(d \varphi^{-1}\right) \circ \varphi=(d \varphi)^{-1}$ which implies that $\operatorname{det}\left(d \varphi^{-1}\right)=[\operatorname{det}(d \varphi)]^{-1} \circ \varphi^{-1}$. Hence, since $d \varphi(t)=\Phi^{t}$, the $L^{2}$-transpose $R_{\varphi(t)}^{\dagger}$ of the right translation operator $R_{\varphi(t)}$ is given by the composition of multiplication by $\left[\operatorname{det} \Phi^{t}\right]^{-1}$ with $R_{\varphi(t)}^{-1}$ i.e.,

$$
R_{\varphi(t)}^{\dagger}=R_{\varphi(t)}^{-1} \circ\left[\operatorname{det} \Phi^{t}\right]^{-1} .
$$

Combining this with item (i) we get

$$
\begin{aligned}
\left(\left(\Phi^{t}\right)^{-1} \circ R_{\varphi(t)}\right)^{\dagger} & =\left(R_{\varphi(t)}^{-1} \circ\left[\operatorname{det} \Phi^{t}\right]^{-1}\right) \circ\left(\left(\Phi^{t}\right)^{-1}\right)^{\dagger} \\
& =R_{\varphi(t)}^{-1} \circ\left(\left[\left(\operatorname{det} \Phi^{t}\right) \Phi^{t}\right]^{-1}\right)^{\dagger} \\
& =R_{\varphi(t)}^{-1} \circ \Psi^{t} .
\end{aligned}
$$

Clearly, $(i)$ and (ii) extend by continuity to negative Sobolev spaces.

Lemma B.2. Assume that $\ell \geq 3, \varphi \in C^{1}\left((-2,2), \mathcal{D}_{+}^{\ell}\right)$, and consider the oneparameter family of differential operators on the torus $\mathbb{T}^{2}$,

$$
\mathcal{P}_{\lambda}(s):=\lambda-\sum_{1 \leq i, j \leq 2} p_{i j}(s) \partial_{x_{i}} \partial_{x_{j}} \quad(-2<s<2),
$$


with coefficients $\left(p_{i j}(s, x)\right)_{1<i, j \leq 2}:=\left(d_{\varphi^{-1}(s, x)} \varphi(s)\right)\left(d_{\varphi^{-1}(s, x)} \varphi(s)\right)^{\dagger}, x \in \mathbb{T}^{2}$. Then, there exists $\lambda_{0} \geq 1$ and $C>0$ so that for any $\lambda \geq \lambda_{0}, 0 \leq s \leq 1$, and $u \in H^{2}\left(\mathbb{T}^{2}, \mathbb{R}^{2}\right)$,

$$
\left\|\mathcal{P}_{\lambda}(s) u\right\|_{L^{2}} \geq C\|u\|_{H^{2}} .
$$

Proof. Let $\lambda=1+\tilde{\lambda}$ and $\tilde{\lambda} \geq 0$. Then for any $u \in H^{2}\left(\mathbb{T}^{2}, \mathbb{R}^{2}\right)$ one has

$$
\left\|\mathcal{P}_{\lambda} u\right\|_{L^{2}}^{2}=\left\|\mathcal{P}_{1} u\right\|_{L^{2}}^{2}+2 \tilde{\lambda} \int_{\mathbb{T}^{2}} \mathcal{P} u \cdot u d x+\left(2 \tilde{\lambda}+\tilde{\lambda}^{2}\right)\|u\|_{L^{2}}^{2}
$$

where $\mathcal{P}:=\left.\mathcal{P}_{\lambda}\right|_{\lambda=0}$, and the dot product denotes the Euclidean scalar product in $\mathbb{R}^{2}$. It follows from the formulas for the coefficients $p_{i j}(s, x)$ that there exists a constant $C_{0}>0$ so that for any $0 \leq s \leq 1, x$ in $\mathbb{T}^{2}$, and $\xi$ in $\mathbb{R}^{2}$,

$$
\sum_{1 \leq i, j \leq 2} p_{i j}(s, x) \xi_{i} \xi_{j} \geq C_{0}\|\xi\|^{2}
$$

and hence

$$
\sum_{1 \leq i, j \leq 2} p_{i j}(s) \partial_{x_{i}} u \cdot \partial_{x_{j}} u \geq C_{0}\left(\sum_{i=1}^{2}\left|\partial_{x_{i}} u_{1}\right|^{2}+\sum_{i=1}^{2}\left|\partial_{x_{i}} u_{2}\right|^{2}\right)
$$

where $u_{1}$ and $u_{2}$ are the components of $u$. Using (B.3) in combination with the latter estimate, we get that there exists $C_{1}>0$ such that for any $\tilde{\lambda} \geq 0,0 \leq s \leq 1$, and $u \in H^{2}\left(\mathbb{T}^{2}, \mathbb{R}^{2}\right)$,

$$
\begin{aligned}
\left\|\mathcal{P}_{\lambda} u\right\|_{L^{2}}^{2} & =\left\|\mathcal{P}_{1} u\right\|_{L^{2}}^{2}+\left(2 \tilde{\lambda}+\tilde{\lambda}^{2}\right)\|u\|_{L^{2}}^{2} \\
& +2 \tilde{\lambda} \int_{\mathbb{T}^{2}} \sum_{1 \leq i, j \leq 2}\left(p_{i j}(s) \partial_{x_{i}} u \cdot \partial_{x_{j}} u-\frac{1}{2}\left(\partial_{x_{i}} \partial_{x_{j}} p_{i j}(s)\right) u \cdot u\right) d x \\
& \geq\left\|\mathcal{P}_{1} u\right\|_{L^{2}}^{2}+\left(\tilde{\lambda}^{2}-C_{1} \tilde{\lambda}\right)\|u\|_{L^{2}}^{2} .
\end{aligned}
$$

Choosing $\tilde{\lambda}_{0} \geq 2 C_{1}$ we obtain that for any $\tilde{\lambda} \geq \tilde{\lambda}_{0}, 0 \leq s \leq 1$, and $u \in H^{2}\left(\mathbb{T}^{2}, \mathbb{R}^{2}\right)$,

$$
\left\|\mathcal{P}_{\lambda} u\right\|_{L^{2}}^{2} \geq\left\|\mathcal{P}_{1} u\right\|_{L^{2}}^{2}+\tilde{\lambda}^{2}\|u\|_{L^{2}}^{2} / 2 .
$$

Note that by (6.5),

$$
\mathcal{P}_{1} u=R_{\varphi^{-1}} \circ(1-\Delta) \circ R_{\varphi} u+d u\left(R_{\varphi^{-1}} \circ \Delta(\varphi)\right) .
$$

Hence there exist positive constants so that for any $0 \leq s \leq 1$ and $u \in H^{2}\left(\mathbb{T}^{2}, \mathbb{R}^{2}\right)$

$$
\begin{aligned}
\left\|\mathcal{P}_{1} u\right\|_{L^{2}}^{2} & \geq\left\|R_{\varphi^{-1}} \circ(1-\Delta) \circ R_{\varphi} u\right\|_{L^{2}}^{2} \\
& +2\left\langle R_{\varphi^{-1}} \circ(1-\Delta) \circ R_{\varphi} u, d u\left(R_{\varphi^{-1}} \circ \Delta(\varphi)\right)\right\rangle_{L^{2}} \\
& \geq C_{2}\|u\|_{H^{2}}^{2}-2\left\|R_{\varphi^{-1}} \circ(1-\Delta) \circ R_{\varphi} u\right\|_{L^{2}} \| d u\left(R_{\varphi^{-1}} \circ \Delta(\varphi) \|_{L^{2}}\right. \\
& \geq C_{2}\|u\|_{H^{2}}^{2}-C_{3}\|u\|_{H^{2}}\|u\|_{H^{1}} \\
& \geq \frac{C_{2}}{2}\|u\|_{H^{2}}^{2}-C_{4}\|u\|_{H^{1}}^{2} \\
& \geq \frac{C_{2}}{4}\|u\|_{H^{2}}^{2}-C_{5}\|u\|_{L^{2}}^{2},
\end{aligned}
$$


where for the latter inequality we have used that, by interpolation, $\|u\|_{H^{1}} \leq$ $C_{6}\|u\|_{H^{2}}^{1 / 2}\|u\|_{L^{2}}^{1 / 2}$, and hence $\|u\|_{H^{1}}^{2} \leq \epsilon\|u\|_{H^{2}}^{2}+C_{6}^{4}\|u\|_{L^{2}}^{2} / \epsilon$ for any $\epsilon>0$. Thus

$$
\left\|\mathcal{P}_{1} u\right\|_{L^{2}}^{2}+\tilde{\lambda}^{2}\|u\|_{L^{2}}^{2} / 2 \geq \frac{C_{2}}{4}\|u\|_{H^{2}}^{2}+\left(\frac{\tilde{\lambda}^{2}}{2}-C_{5}\right)\|u\|_{L^{2}}^{2} .
$$

Together with (B.4) this implies that for $\tilde{\lambda}_{0}=\max \left\{2 C_{1}, \sqrt{2 C_{5}}\right\}$ and $C=\sqrt{C_{2}} / 2>0$ one has that for any $\tilde{\lambda} \geq \tilde{\lambda}_{0}, 0 \leq s \leq 1$, and $u \in H^{2}\left(\mathbb{T}^{2}, \mathbb{R}^{2}\right)$,

$$
\left\|\mathcal{P}_{\lambda}(s) u\right\|_{L^{2}} \geq C\|u\|_{H^{2}} .
$$

$\square$

The following result is well known. For the convenience of the reader we include an elementary proof for it.

Lemma B.3. Let $k \geq 1$ and $s \geq k+2$. Then, for any $k-s \leq m \leq s-1$ and for any real-valued functions $f \in H^{s}$ and $g \in H^{m},\left[A_{k}^{1 / 2}, f\right] g:=A_{k}^{1 / 2}(f g)-f A_{k}^{1 / 2} g$ is in $H^{m-k+1}$ and the bilinear mapping

$$
H^{s} \times H^{m} \rightarrow H^{m-k+1},(f, g) \mapsto\left[A_{k}^{1 / 2}, f\right] g
$$

is continuous.

Proof. Consider the first order operator,

$$
P_{1}:=\left(A_{k}\right)^{\frac{1}{2 k}} .
$$

Then the complete symbol of $P_{1}$ is the Fourier multiplier

$$
\sigma_{1}(\xi)=\left(\sum_{j=0}^{k}(2 \pi)^{2 j}\left(\xi_{1}^{2}+\xi_{2}^{2}\right)^{j}\right)^{\frac{1}{2 k}} .
$$

It means that for any $\mathbb{Z}^{2}$-periodic distribution $h \in \mathcal{D}^{\prime}\left(\mathbb{T}^{2}\right)$ the Fourier coefficients $\widehat{\left(P_{1} h\right)}(\xi), \xi \in \mathbb{Z}^{2}$, of $P_{1} h$ are given by $\widehat{\left(P_{1} h\right)}(\xi)=\sigma_{1}(\xi) \hat{h}(\xi)$. Clearly, for any $l \in \mathbb{Z}$, $P_{1}: H^{l} \rightarrow H^{l-1}$ is a bounded linear operator. Assume that $|m| \leq s-1$. For any $f \in H^{s}$ and $g \in H^{m}$ we have for $\xi \in \mathbb{Z}^{2}$,

$$
\begin{aligned}
\mid\left(\left[\widehat{\left.P_{1}, f\right] g}\right)(\xi) \mid\right. & =\left|\widehat{P_{1}(f g)}(\xi)-\widehat{f P_{1} g}(\xi)\right| \\
& \leq\left|\sigma_{1}(\xi) \sum_{\eta \in \mathbb{Z}^{2}} \hat{f}(\xi-\eta) \hat{g}(\eta)-\sum_{\eta \in \mathbb{Z}^{2}} \hat{f}(\xi-\eta) \sigma_{1}(\eta) \hat{g}(\eta)\right| \\
& \leq \sum_{\eta \in \mathbb{Z}^{2}}\left|\sigma_{1}(\xi)-\sigma_{1}(\eta)\right||\hat{f}(\xi-\eta)||\hat{g}(\eta)| \\
& \leq C_{k} \sum_{\eta \in \mathbb{Z}^{2}}(1+|\xi-\eta|)|\hat{f}(\xi-\eta)||\hat{g}(\eta)|
\end{aligned}
$$

where for the latter inequality we used that, for some constant $C_{k}>0$,

$$
\left|\sigma_{1}(\xi)-\sigma_{1}(\eta)\right| \leq C_{k}(1+|\xi-\eta|) \quad \forall \xi, \eta \in \mathbb{Z}^{2} .
$$

It follows from (B.7) and Lemma B.4 below that there exists $C_{k}>0$ such that for any $|m| \leq s-1$, and for any $f \in H^{s}$ and $g \in H^{m}$,

$$
\left\|\left[P_{1}, f\right] g\right\|_{H^{m}} \leq C_{k}\|f\|_{H^{s}}\|g\|_{H^{m}} .
$$


On the other side, as $A_{k}^{1 / 2}=P_{1}^{k}$, we have for any $f \in H^{s}$ and $g \in H^{m}$ with $k-s \leq$ $m \leq s-1$

$$
\left[A_{k}^{1 / 2}, f\right] g=\left[P_{1}, f\right] P_{1}^{k-1} g+P_{1}\left[P_{1}, f\right] P_{1}^{k-2} g+\ldots+P_{1}^{k-1}\left[P_{1}, f\right] g
$$

which together with (B.8) implies the statement of the lemma.

In the proof of Lemma B.3 we used the following well-known fact. Denote by $h^{l} \equiv h^{l}\left(\mathbb{Z}^{2}, \mathbb{R}\right)$ the Hilbert space

$$
h^{l}=\left\{a=(a(\xi))_{\xi \in \mathbb{Z}^{2}} \mid\|a\|_{h^{l}}<\infty, a(-\xi)=a(\xi) \forall \xi \in \mathbb{Z}^{2}\right\}
$$

where

$$
\|a\|_{h^{l}}:=\left(\sum_{\xi \in \mathbb{Z}^{2}}(1+\|\xi\|)^{2 l}|a(\xi)|^{2}\right)^{1 / 2}
$$

LEMmA B.4. Let $s \geq 3$ and $|m| \leq s$. Then the convolution

$$
h^{s} \times h^{m} \rightarrow h^{m}, \quad(a, b) \mapsto(a * b)(\xi):=\sum_{\eta \in \mathbb{Z}^{2}} a(\xi-\eta) b(\eta)
$$

is continuous.

The proof of the lemma is straightforward and we omit it.

REMARK B.5. In the proof of Proposition 5.1, we use Lemma B.3 as follows. First let us recall the set-up. Let $\ell \geq \ell_{k}=2 k+5$ be arbitrary and assume that the solution curve $(\varphi, v)$ of (1.2) with initial data $\left(\mathrm{id}, v_{0}\right)$ and $v_{0}$ in $H^{\ell}$, is in $C^{1}\left((-2,2), \mathcal{D}_{+}^{\ell} \times\right.$ $\left.H^{\ell}\right)$. Then, $u=v \circ \varphi^{-1}$ is a solution of $(1.7)$ in $C^{0}\left((-2,2), H^{\ell}\right) \cap C^{1}\left((-2,2), H^{\ell-1}\right)$. Further, $\mathcal{P}_{\lambda}^{\dagger} \mathcal{P}_{\lambda}$, with $\mathcal{P}_{\lambda}$ given by (6.7), is a fourth order differential operator with coefficients in $C^{0}\left((-2,2), H^{\ell-3}\right)$. Up to lower order, $\mathcal{P}_{\lambda}^{\dagger} \mathcal{P}_{\lambda}$ is equal to $\sum_{|\alpha|=4} f_{\alpha} \partial^{\alpha}$, where the coefficients $f_{\alpha}$ are in $C^{0}\left((-2,2), H^{\ell-3}\right)$ (in fact they take values in $H^{\ell-1}$ ). This shows that for any $h$ in $H^{\ell}$,

$$
\left[A_{k}^{1 / 2}, \mathcal{P}_{\lambda}^{\dagger} \mathcal{P}_{\lambda}\right] h=A_{k}^{1 / 2} \sum_{|\alpha|=4} f_{\alpha} \partial^{\alpha} h-\sum_{|\alpha|=4} f_{\alpha} A_{k}^{1 / 2}\left(\partial^{\alpha} h\right)+\ldots
$$

where ... stand for terms in $C^{0}\left((-2,2), H^{\ell-k-3}\right)$. Moreover, the assumptions above imply that, for any $-2<s<2$, each of the entries of the fundamental matrix solutions $\Phi^{s}$ and $\Psi^{s}$ of (6.1), respectively, (6.3) is a continuous function of $t$ with values in $H^{\ell-1}$. At this point it is crucial that we assume that $k \geq 1$. It guarantees that $A_{k}^{-1} \circ R_{\varphi(s)}^{-1} \circ \Psi^{s}\left(A_{k} v_{0}\right)$ is in $C^{0}\left((-2,2), H^{\ell}\right)$. Thus, for any $\alpha \in \mathbb{Z}_{\geq 0}^{2}$ with $|\alpha|=4$, it follows that $g_{\alpha}:=\partial^{\alpha}\left(A_{k}^{-1} \circ R_{\varphi(s)}^{-1} \circ \Psi^{s}\left(A_{k} v_{0}\right)\right) \in C^{0}\left((-2,2), H^{\ell-4}\right)$. Hence, by Lemma B.3 applied to $f_{\alpha} \in H^{\ell-3}$ and $g_{\alpha} \in H^{\ell-4}$ for any $\alpha \in \mathbb{Z}_{\geq 0}^{2}$ with $|\alpha|=4$,

$$
\left[A_{k}^{1 / 2}, \mathcal{P}_{\lambda}^{\dagger} \mathcal{P}_{\lambda}\right] \circ A_{k}^{-1} \circ R_{\varphi(s)}^{-1} \circ \Psi^{s}\left(A_{k} v_{0}\right) \in C^{0}\left((-2,2), H^{\ell-k-3}\right) .
$$

As a consequence,

$$
\Phi^{t} \int_{0}^{t}\left(\Phi^{s}\right)^{-1} \circ R_{\varphi(s)} \circ A_{k}^{-1 / 2} \circ\left[A_{k}^{1 / 2}, \mathcal{P}_{\lambda}^{\dagger}(s) \mathcal{P}_{\lambda}(s)\right] \circ A_{k}^{-1} \circ R_{\varphi(s)}^{-1} \circ \Psi^{s} d s\left(A_{k} v_{0}\right)
$$


is in $C^{0}\left((-2,2), H^{\ell-3}\right)$.

Appendix C. Euler-Lagrange equation. For the convenience of the reader we review in this appendix the derivation of the Euler-Lagrange equation of the action functional $\mathcal{E}_{k}^{T}$ induced by the right-invariant, weak Riemannian metric $\nu^{(k)}$ on the group $\mathcal{D}_{+}$for any $k \geq 0$. Recall that the $H^{k}$-Sobolev type inner product is given by

$$
\langle u, v\rangle_{k}:=\int_{\mathbb{T}^{2}}\left\langle u, A_{k} v\right\rangle d x, \quad \forall u, v \in T_{\mathrm{id}} \mathcal{D}_{+}
$$

where $\langle\cdot, \cdot\rangle$ denotes the Euclidean scalar product in $\mathbb{R}^{2}$, and

$$
A_{k}:=\mathrm{Id}+\sum_{j=1}^{k}(-\Delta)^{j}
$$

It induces the right-invariant metric $\nu^{(k)}$ on $\mathcal{D}_{+}$which, at any $\varphi$ in $\mathcal{D}_{+}$, is defined by

$$
\nu_{\varphi}^{(k)}(\xi, \eta):=\left\langle\left(d_{\mathrm{id}} R_{\varphi}\right)^{-1} \xi,\left(d_{\mathrm{id}} R_{\varphi}\right)^{-1} \eta\right\rangle_{k}, \quad \forall \xi, \eta \in T_{\varphi} \mathcal{D}_{+}
$$

where $R_{\varphi}: \mathcal{D}_{+} \rightarrow \mathcal{D}_{+}, \psi \mapsto \psi \circ \varphi$ denotes the right translation by $\varphi$. Note that $d_{\mathrm{id}} R_{\varphi}: T_{\mathrm{id}} \mathcal{D}_{+} \rightarrow T_{\varphi} \mathcal{D}_{+}$is given by $\left(d_{\mathrm{id}} R_{\varphi}\right) u=u \circ \varphi$. In particular, for any curve $\gamma:[0, T] \rightarrow \mathcal{D}_{+}, t \mapsto \gamma(t)$, emanating from the identity,

$$
\left(d_{\mathrm{id}} R_{\gamma(t)}\right)^{-1} \dot{\gamma}(t)=\dot{\gamma}(t) \circ \gamma^{-1}(t)
$$

The action functional $\mathcal{E}_{k}^{T}$ induced by $\nu^{(k)}$ on the space of $C_{F}^{2}$-curves is given by $(1.1 \mathrm{~b})$ i.e.,

$$
\mathcal{E}_{k}^{T}(\gamma)=\frac{1}{2} \int_{0}^{T} \int_{\mathbb{T}^{2}}\left\langle\dot{\gamma}(t) \circ \gamma^{-1}(t), A_{k}\left(\dot{\gamma}(t) \circ \gamma^{-1}(t)\right)\right\rangle d t d x
$$

To derive the Euler-Lagrange equation of (C.1), let $\gamma:(-\varepsilon, \varepsilon) \times[0, T] \rightarrow \mathcal{D}_{+},(s, t) \mapsto$ $\gamma(s, t)$ be a $C_{F}^{2}$-smooth variation of the $C_{F}^{2}$-smooth curve $\varphi:[0, T] \rightarrow \mathcal{D}_{+}, t \mapsto$ $\gamma(0, t):=\varphi(t)$ with fixed end points

$$
\gamma(s, 0) \equiv \mathrm{id} ; \gamma(s, T) \equiv \varphi(T), \quad \text { for every } \quad-\varepsilon<s<\varepsilon .
$$

For clarity of exposition, we will omit the explicit time and/or parameter dependence, e.g. we will write $\gamma$ and $\varphi$ instead of $\gamma(s, t)$ and $\varphi(t)$ respectively, and so on.

As $A_{k}$ is symmetric, it follows from (C.1) that

$$
\begin{aligned}
\delta \mathcal{E}_{k}^{T}(\varphi) & :=\left.\frac{d}{d s}\right|_{s=0} \mathcal{E}_{k}^{T}(\gamma) \\
& =\int_{0}^{T} \int_{\mathbb{T}^{2}}\left\langle\delta\left(\dot{\varphi} \circ \varphi^{-1}\right), A_{k}\left(\dot{\varphi} \circ \varphi^{-1}\right)\right\rangle d t d x
\end{aligned}
$$

By the chain rule we have

$$
\delta\left(\dot{\varphi} \circ \varphi^{-1}\right)=(\delta \varphi)^{\circ} \circ \varphi^{-1}+\left[(d \dot{\varphi}) \circ \varphi^{-1}\right] \delta \varphi^{-1} .
$$


The variation of the inverse diffeomorphism $\delta \varphi^{-1}$ may be expressed in terms of $\delta \varphi$ by an application of the chain rule to the identity $\varphi \circ \varphi^{-1}=\mathrm{Id}$,

$$
\delta \varphi^{-1}=-\left[(d \varphi)^{-1} \delta \varphi\right] \circ \varphi^{-1}
$$

where $(d \varphi)^{-1}$ is the section $x \mapsto(d \varphi)^{-1}(x)=\left(d_{x} \varphi\right)^{-1}$. Hence

$$
\delta\left(\dot{\varphi} \circ \varphi^{-1}\right)=\left[(\delta \varphi)^{\cdot}-(d \dot{\varphi})(d \varphi)^{-1} \delta \varphi\right] \circ \varphi^{-1} .
$$

Similar arguments show that

$$
d\left(\dot{\varphi} \circ \varphi^{-1}\right)=-\left[(d \dot{\varphi})(d \varphi)^{-1}\right] \circ \varphi^{-1} .
$$

Hence, with

$$
u:=\dot{\varphi} \circ \varphi^{-1}
$$

one gets

$$
\delta u=\delta\left(\dot{\varphi} \circ \varphi^{-1}\right)=\left\{(\delta \varphi)^{\cdot}-[(d u) \circ \varphi] \delta \varphi\right\} \circ \varphi^{-1} .
$$

Substituting the latter identity into formula (C.3), yields

$$
\begin{aligned}
\delta \mathcal{E}_{k}^{T}(\varphi) & =\int_{0}^{T} \int_{\mathbb{T}^{2}}\left\langle\left\{(\delta \varphi)^{\cdot}-[(d u) \circ \varphi] \delta \varphi\right\} \circ \varphi^{-1}, A_{k} u\right\rangle d t d x \\
& =\int_{0}^{T} \int_{\mathbb{T}^{2}}\left\langle(\delta \varphi)^{\cdot}-[(d u) \circ \varphi] \delta \varphi,\left(A_{k} u\right) \circ \varphi\right\rangle \operatorname{det}(d \varphi) d t d y
\end{aligned}
$$

where the latter identity results from the change of variables $x=\varphi(t, y)$. Clearly, $\left\langle[(d u) \circ \varphi] \delta \varphi,\left(A_{k} u\right) \circ \varphi\right\rangle=\left\langle\delta \varphi,\left[(d u)^{\dagger}\left(A_{k} u\right)\right] \circ \varphi\right\rangle$. Hence, upon integrating by parts with respect to $t$ keeping in mind that the end points of the curves $\gamma$ are held fixed (see (C.2)), we get

$$
\delta \mathcal{E}_{k}^{T}(\varphi)=-\int_{0}^{T} \int_{\mathbb{T}^{2}}\left\langle\delta \varphi,\left\{\operatorname{det}(d \varphi)\left[\left(A_{k} u\right) \circ \varphi\right]\right\}^{\cdot}+\operatorname{det}(d \varphi)\left(\left[(d u)^{\dagger} A_{k} u\right] \circ \varphi\right)\right\rangle d t d x .
$$

It follows that the critical points $\varphi$ of the action functional satisfy the Euler-Lagrange equation

$$
\left\{\operatorname{det}(d \varphi)\left[\left(A_{k} u\right) \circ \varphi\right]\right\}^{\cdot}+\operatorname{det}(d \varphi)\left(\left[(d u)^{\dagger} A_{k} u\right] \circ \varphi\right)=0 .
$$

This equation can be simplified as follows. Using the well-known identity relating determinant and trace of any one parameter family of regular square matrices depending smoothly on the parameter,

$$
[\log (\operatorname{det}(d \varphi))]^{\cdot}=\operatorname{Tr}\left[(d \dot{\varphi})(d \varphi)^{-1}\right]=\operatorname{Tr}[(d u) \circ \varphi]=(\operatorname{div} u) \circ \varphi,
$$

one gets

$$
\left\{\operatorname{det}(d \varphi)\left[\left(A_{k} u\right) \circ \varphi\right]\right\}^{*}=\operatorname{det}(d \varphi)\left(\left[A_{k} \dot{u}+\left(d A_{k} u\right) u+\operatorname{div} u \cdot A_{k} u\right] \circ \varphi\right) .
$$

Thus (C.4) reads

$$
\operatorname{det}(d \varphi)\left(\left[A_{k} \dot{u}+\left(d A_{k} u\right) u+\left(\operatorname{div} u \cdot \operatorname{Id}+(d u)^{\dagger}\right) A_{k} u\right] \circ \varphi\right)=0 .
$$


Finally, since the factor $\operatorname{det}(d \varphi)$ is everywhere positive, we can drop it from the latter identity. Moreover if we factor out the right translation $R_{\varphi}$, we end up with the following equivalent more compact formulation of (C.4):

$$
A_{k} \dot{u}+\left(d A_{k} u\right) u+\left(\operatorname{div} u \cdot \operatorname{Id}+(d u)^{\dagger}\right) A_{k} u=0 .
$$

We remark that (C.5) is a transport equation for $A_{k} u$. Indeed, upon observing that $\left(d A_{k} u\right) u=(u \cdot \nabla) A_{k} u$, where $u \cdot \nabla$ is the vector field $u_{1} \partial_{x_{1}}+u_{2} \partial_{x_{2}}$ acting componentwise, it reads

$$
\left(A_{k} u\right)^{\cdot}+\left[(u \cdot \nabla)+\operatorname{div} u \cdot \operatorname{Id}+(d u)^{\dagger}\right] A_{k} u=0
$$

\section{REFERENCES}

[1] V. ARnold, Sur la géometrie differentielle des groupes de Lie de dimension infinie et ses applications à l'hydrodynamique des fluids parfaits, Ann. Inst. Fourier, 16, (1966), pp. 319 361.

[2] V. Arnold and B. Khesin, Topological methods in hydrodynamics, Springer, 1998.

[3] J. Burgers, A mathematical model illustrating the theory of turbulence, Adv. Appl. Mech., 1 (1948), pp. 171-199.

[4] R. Camassa And D. Holm, An integrable shallow water equation with peaked solitons, Phys. Rev. Lett, 71 (1993), pp. 1661-1664.

[5] A. Constantin And J. Escher, Well-posedness, global existence, and blow-up phenomena for a periodic quasi-linear hyperbolic equation, Commun. Pure Appl. Math., 51 (1998), pp. $475-504$.

[6] A. Constantin, T. Kappeler, B. Kolev and P. Topalov, On geodesic exponential maps of the Virasoro group, Ann. Glob. Anal. Geom., 31 (2007), pp. 155-180.

[7] A. Constantin And B. Kolev, On the geometric approach to the motion of inertial mechanical systems, J. Phys. A, 35 (2002), pp. R51-R79.

[8] A. Constantin and B. Kolev, Geodesic flow on the diffeomorphism group of the circle, Comment. Math. Helv., 78 (2003), pp. 787-804.

[9] A. Constantin and H. McKean, A shallow water equation on the circle, Commun. Pure Appl. Math., 52 (1999), pp. 949-982.

[10] C. De Lellis, T. KAppeler AND P. TOpalov, Low-regularity solutions of the periodic Camassa-Holm equation, Comm. in PDE, 32 (2007), pp. 87-126.

[11] D. EBIn, The manifold of riemannian metrics, in Global Analysis, Proc. Sympos. Pure Math., vol 15, Amer. Math. Soc., eds. S.S. Chern and S. Smale, 11-40, and Bull. Amer. Math. Soc., 74 (1968), pp. 1001-1003.

[12] D. EBin And J. MARSDEn, Groups of diffeomorphisms and the motion of an incompressible fluid, Ann. Math., 92 (1970), pp. 102-163.

[13] A. FokAs AND B. FuchssteineR, Symplectic structures, their Bäcklund transformation and hereditary symmetries, Physica D, 4 (1981), pp. 47-66.

[14] R. Hamilton, The inverse function theorem of Nash and Moser, Bull. Amer. Math. Soc., 7 (1982), pp. 66-222.

[15] D. Holm, J. Marsden, T. Ratiu and S. Shkoller, The Euler-Poincaré equations and semidirect products with applications to continuum theories, Adv. Math., 137 (1998), pp. 1-81.

[16] T. Kappeler, E. Loubet and P. Topalov, Analyticity of Riemannian exponential maps on $\operatorname{Diff}(\mathbb{T})$, J. of Lie Theory 17 (2007), pp. 481-503.

[17] T. Kappeler and J. PÖschel, KdVEKKAM, Springer-Verlag, Berlin, 2003.

[18] T. Kaто, The Cauchy problem for quasi-linear symmetric hyperbolic systems, Arch. Ration. Mech. Anal., 58 (1975), pp. 181-205.

[19] B. KHESin AND G. MisioleK, Euler equations on homogeneous spaces and Virasoro orbits, Adv. Math, 176 (2003), pp. 116-144.

[20] S. Kouranbaeva, The Camassa-Holm equation as a geodesic flow on the diffeomorphism group, J. Math. Phys., 40 (1999), pp. 857-868.

[21] A. Kriegl and P. Michor, The Convenient Setting of Global Analysis, AMS Series: Mathematical surveys and monographs, Vol. 53, 1997.

[22] S. LAng, Differential manifolds, Addison-Wesley Series in Mathematics, 1972.

[23] P. LAx, Functional Analysis, Wiley, 2002. 
[24] J. Milnor, Remarks on infinite-dimensional Lie groups, Les Houches, Session XL, 1983, Elsevier Science Publishers B.V., 1984.

[25] G. MisioleK, A shallow water equation as a geodesic flow on the Bott-Virasoro group, J. Geom. Phys., 24 (1998), pp. 203-208.

[26] G. MisioleK, Classical solutions of the periodic Camassa-Holm equation, GAFA, 12 (2002), pp. 1080-1104.

[27] H. OMORI, On the group of diffeomorphisms on a compact manifold, in Global Analysis, Proc. Sympos. Pure Math., 15, eds. S.S. Chern and S.Smale, Amer. Math. Soc., pp. 167-183.

[28] R. PALais, Foundations of global non-linear analysis, Benjamin, N.Y. 1968.

[29] R. PAlais, Seminar on the Atiyah-Singer index theorem, Princeton, 1965. 